\author{
Universidade Federal do Rio Grande do Norte \\ Centro de Tecnologia \\ Programa de Pós-Graduação em Engenharia Elétrica
}

\title{
Uma Contribuição à Solução do Problema dos k-Servos Usando Aprendizagem por Reforço
}

$\begin{array}{ll}\text { Autor: } & \text { Manoel Leandro de Lima Júnior } \\ \text { Orientador: } & \text { Prof. Dr. Jorge Dantas de Melo } \\ \text { Co-orientador: } & \text { Prof. Dr. Adrião Dória Duarte Neto }\end{array}$

Natal, Abril de 2005 
Universidade Federal do Rio Grande do Norte

Centro de Tecnologia

Programa de Pós-Graduação em Engenharia Elétrica

\author{
Manoel Leandro de Lima Júnior
}

\title{
Uma Contribuição à Solução do Problema dos k-Servos Usando Aprendizagem por Reforço
}

Dissertação submetida ao Programa de Pós-

Graduação em Engenharia Elétrica do Centro de Tecnologia da Universidade Federal do Rio Grande do Norte, como parte dos requisitos necessários para obtenção do grau de Mestre em Ciências (M.Sc.).

Natal, Abril de 2005 


\section{Universidade Federal do Rio Grande do Norte Centro de Tecnologia \\ Programa de Pós-Graduação em Engenharia Elétrica}

Aprovada em 06 de Abril de 2005 pela comissão examinadora, formada pelos seguintes membros:

Prof. D.Sc. Jorge Dantas de Melo (Orientador) DCA - UFRN

Prof. D.Sc. Adrião Duarte Dória Neto (Co-Orientador) DCA - UFRN

Prof. D.Sc. Weber Martins (Examinador Externo) EEE - UFG

Prof. D.Sc. Dario José Aloise (Primeiro Examinador Interno)

Dimap - UFRN

Prof. D.Sc. Manoel Firmino de Medeiros Júnior (Segundo Examinador Interno) DCA - UFRN 


\section{Resumo}

Neste trabalho é proposto um novo algoritmo online para o resolver o Problema dos $k$-Servos (PKS). O desempenho desta solução é comparado com o de outros algoritmos existentes na literatura, a saber, os algoritmos Harmonic e Work Function ([22] e [23]), que mostraram ser competitivos, tornando-os parâmetros de comparação significativos. Um algoritmo que apresente desempenho eficiente em relação aos mesmos tende a ser competitivo também, devendo, obviamente, se provar o referido fato. Tal prova, entretanto, foge aos objetivos do presente trabalho.

O algoritmo apresentado para a solução do PKS é baseado em técnicas de aprendizagem por reforço. Para tanto, o problema foi modelado como um processo de decisão em múltiplas etapas, ao qual é aplicado o algoritmo $Q$ Learning, um dos métodos de solução mais populares para o estabelecimento de políticas ótimas neste tipo de problema de decisão.

Entretanto, deve-se observar que a dimensão da estrutura de armazenamento utilizada pela aprendizagem por reforço para se obter a política ótima cresce em função do número de estados e de ações, que por sua vez é proporcional ao número $n$ de nós e $k$ de servos. Ao se analisar esse crescimento (matematicamente, $\mathcal{C}_{n, k} \simeq \mathcal{O}\left(n^{k}\right)$ ) percebe-se que o mesmo ocorre de maneira 
exponencial, limitando a aplicação do método a problemas de menor porte, onde o número de nós e de servos é reduzido. Este problema, denominado maldição da dimensionalidade, foi introduzido por Belmann [9] e implica na impossibilidade de execução de um algoritmo para certas instâncias de um problema pelo esgotamento de recursos computacionais para obtenção de sua saída.

De modo a evitar que a solução proposta, baseada exclusivamente na aprendizagem por reforço, seja restrita a aplicações de menor porte, propõese uma solução alternativa para problemas mais realistas, que envolvam um número maior de nós e de servos. Esta solução alternativa é hierarquizada e utiliza dois métodos de solução do PKS: a aprendizagem por reforço, aplicada a um número reduzido de nós obtidos a partir de um processo de agregação, e um método guloso, aplicado aos subconjuntos de nós resultantes do processo de agregação, onde o critério de escolha do agendamento dos servos é baseado na menor distância ao local de demanda. 


\section{Sumário}

1 Introdução 1

2 Computação Online e Análise Competitiva $\quad 6$

2.1 Introdução . . . . . . . . . . . . . . . . . . . 6

2.2 Problemas de Otimização Online e Offline . . . . . . . . . . 7

2.3 Análise Competitiva: Competitividade e Taxa de Competitividade ........................ 10

3 Problema dos k-Servos 13

3.1 Introdução . . . . . . . . . . . . . . . . . 13

3.2 Conceitos Preliminares . . . . . . . . . . . . . . 14

3.2.1 Espaço Métrico . . . . . . . . . . . . . . . 14

3.2.2 Definição Formal do PKS . . . . . . . . . . . . 15

3.3 Estratégias de Solução . . . . . . . . . . . . . . . 16

3.3.1 Algoritmos Heurísticos . . . . . . . . . . . . 16

3.3.2 Algoritmo Harmonic . . . . . . . . . . . . . . . . 20

3.3.3 Algoritmo Work Function (WFA) - Função de Trabalho 22

3.4 Aplicações . . . . . . . . . . . . . . . . . . . . . . . . . . 30 
4 Aprendizagem por Reforço 32

4.1 Introdução . . . . . . . . . . . . . . . . . . . . . 32

4.2 Principais Características . . . . . . . . . . . 36

4.2.1 Maximização do Retorno Global . . . . . . . . . . . . . 36

4.2.2 Diversificação versus Intensificação . . . . . . . . . . . 37

4.3 Fundamentos matemáticos . . . . . . . . . . . . . . . . . 38

4.3.1 Propriedade de Markov . . . . . . . . . . . . . . 38

4.3.2 Processos de Decisão Markovianos - PDM ..... 39

4.4 Elementos ....................... . . 40

4.4.1 Interface Agente-Ambiente . . . . . . . . . . . 42

4.4 .2 Política ................... . 42

4.4 .3 Reforço e Retorno . . . . . . . . . . . . . . . . . . . . . 42

4.4 .4 Função de Valor . . . . . . . . . . . . . . . . . . . . . . 44

4.5 Métodos ...................... . . 46

4.5.1 Programação Dinâmica (Dynamic Programming - DP) 46

4.5.2 Métodos de Monte Carlo (MC Methods) . . . . . . 47

4.5.3 Método de Diferenças Temporais (Temporal Difference Method - TD) . . . . . . . . . . . . . 48

\section{Aplicação da Aprendizagem por Reforço ao Problema dos} k-Servos $\quad 52$

5.1 Introdução . . . . . . . . . . . . . . . . . . 52

5.2 Representação dos Estados . . . . . . . . . . . . . . . . 53

5.3 Q-Learning Aplicado ao Problema dos k-Servos . . . . . . . 54

5.4 Estrutura de Dados para o Problema . . . . . . . . . . . . 55

5.5 Resultados ................... 56 
6 Solução Hierárquica $\quad 59$

6.1 Introdução . . . . . . . . . . . . . . . . . . . . 59

6.2 Apresentação da Solução Hierárquica . . . . . . . . . . . . . . 61

6.2.1 Divisão em Grupamentos de Proximidade ..... . 62

6.2.2 Seleção dos Nós - Escolha dos Centros dos Grupamentos 64

6.2.3 Execução da Aprendizagem por Reforço no Conjunto Reduzido de Nós . . . . . . . . . . . . . . . . . . 64

6.2.4 Implementação do Algoritmo Hierárquico . . . . . . . . 66

6.3 Complexidade do Algoritmo Hierárquico . . . . . . . . . . 70

6.4 Resultados . . . . . . . . . . . . . . . . 71

$\begin{array}{lll}7 & \text { Considerações Finais } & 76\end{array}$

7.1 Conclusão . . . . . . . . . . . . . . . . . 76

7.2 Perspectivas de Trabalhos Futuros . . . . . . . . . . . . . 78 


\section{Lista de Figuras}

3.1 Algoritmo de Sleator e Tarjan. . . . . . . . . . . . . . 17

3.2 Limitação do algoritmo online de Sleator e Tarjan. .... . . 18

3.3 Algoritmo Guloso. . . . . . . . . . . . . . . . . . . 19

3.4 A estratégia gulosa não garante solução ótima. . . . . . . . . . 19

3.5 Algoritmo Harmonic. . . . . . . . . . . . . . . . . . . 21

3.6 Funcionamento do algoritmo Harmonic. . . . . . . . . . . . . 21

3.7 Grafo. . . . . . . . . . . . . . . . 26

3.8 Algoritmo Work Function. . . . . . . . . . . . . . . . . . . . . 29

4.1 Diagrama esquemático de um sistema de aprendizagem por reforço. . . . . . . . . . . . . . . 35

4.2 Interação entre elementos da aprendizagem por reforço. . . . . 41

4.3 Diagrama de backup do algoritmo Q-Learning. . . . . . . . . . 49

4.4 Algoritmo Q-Learning. . . . . . . . . . . . . 50

5.1 Diagrama de backup com todas as ações possíveis em $s^{\prime}$. . . 55

6.1 Gráfico da dimensão da estrutra de armazenamento da função

$Q$ para alguns valores de $n$ e $k \ldots \ldots$. . . . . . . 60

6.2 Algoritmo de Boruvka. . . . . . . . . . . . . . . 63 
6.3 Algoritmo de seleção dos nós-centro. . . . . . . . . . . . . 65

6.4 Algoritmo Hierárquico. . . . . . . . . . . . . . . . . . . . . 67 


\section{Lista de Tabelas}

3.1 Exemplo de aplicação do algoritmo Work Function. . . . . . . 28

5.1 Estrutura para o armazenamento dos valores de $Q(s, a)$. . . 56

5.2 Resultados obtidos nos testes. . . . . . . . . . . . . . . 58

6.1 Resultados experimentais da comparação entre os algoritmos Hierárquico e Q-Learning. . . . . . . . . . . 73

6.2 Resultados experimentais da comparação entre os algoritmos Hierárquico e Harmonic. . . . . . . . . . . . . . . . . . . . 75 


\title{
Lista de Abreviaturas
}

\author{
AGM - Árvore Geradora Mínima \\ DP - Dynamic Programming - Programação Dinâmica \\ MC - Método de Monte Carlo \\ PKS - Problema dos $k$-Servos \\ PDM - Processos de Decisão Markoviano \\ SO - Sistema Operacional \\ TD - Temporal Difference - Método das Diferenças Temporais \\ WFA - Work Function Algorithm - Algoritmo Função Trabalho
}




\section{Capítulo 1}

\section{Introdução}

O problema dos k-servos (PKS), introduzido por Manasse, McGeoch e Sleator [1], é um problema online com amplas possibilidades de aplicação e bastante estudado na literatura. O mesmo pode ser definido da seguinte forma: dada uma configuração inicial de servos em um grafo $G$ e uma seqüência de requisições $\sigma$, encontre uma política $\pi$ que indique os movimentos dos servos de modo que o custo total dos deslocamentos seja o menor possível. Desde que o atendimento de cada uma das requisições está associada ao deslocamento de um servo, comumente o objetivo a ser alcançado é a minimização da distância total percorrida por todos os servos.

Algoritmos offline de solução do PKS, caracterizados pelo conhecimento a priori da sequiência de requisições, normalmente produzem uma solução ótima. No caso online, não se tem conhecimento sobre as solicitações futuras. Dentro deste contexto, a qualidade de um algoritmo online pode ser quantificada em relação ao desempenho de um algoritmo offline, que possui conhecimento completo das informações futuras. Dentro deste contexto, 
surge o conceito de competitividade, que diz que um algoritmo é c-competitivo se existirem constantes $c$ e $\alpha$, tal que a inequação $\mathcal{C}_{A}(\sigma) \leq c \cdot \mathcal{C}_{O P T}(\sigma)+\alpha$ é válida para qualquer seqüência de requisições $\sigma$. Nessa expressão, $\mathcal{C}_{A}(\sigma)$ e $\mathcal{C}_{O P T}(\sigma)$ correspondem aos custos associados ao algoritmo online $\mathrm{A}$ e ao algoritmo ótimo offline, respectivamente.

Em razão disso e diante da conjectura dos $k$-servos [1], que declara existir um algoritmo ótimo online que é k-competitivo, os pesquisadores vêm tentando há vários anos encontrar soluções que consigam confirmar a veracidade da conjectura mencionada [22]. Por enquanto, esta questão ainda está em aberto (pelo menos ao conhecimento do autor dessa dissertação). Diversos algoritmos já foram propostos, mas nenhum deles conseguiu provar ser $k$ competitivo para todos os casos. Dentre os propostos, dois deles tiveram suas taxas de competitividade provadas para algumas instâncias do PKS e são bastante citados pela literatura: os algoritmos Harmonic e Work Function $([22]$ e $[23])$.

Este trabalho visa propor uma nova solução para o problema dos k-servos através da implementação de um algoritmo original, baseado em técnicas de aprendizagem por reforço. A escolha da mesma ocorreu por causa da generalidade de sua formulação, o que permite a sua aplicação em vários problemas correlatos ao k-servos.

Olhando sob outra ótica o problema dos k-servos, pode-se verificar a adequabilidade da aprendizagem por reforço ao problema em questão. O PKS pode ser visto como um processo de decisão em múltiplas etapas, onde a cada instante $t_{i}(i=1,2, \ldots, m)$ considerado, uma decisão deve ser tomada sobre como atender a solicitação $\sigma_{i}$. Esse processo é também markoviano, uma vez 
que a decisão a ser tomada no instante $t_{i}$ depende apenas das informações disponíveis nesse instante. Dentre as metodologias utilizadas na solução dos problemas de decisão markovianos, uma abordagem bastante eficiente é a aprendizagem por reforço [6]. O objetivo buscado é determinar uma política ótima para a tomada de decisões. Como será explicitado mais adiante, sob o paradigma da aprendizagem por reforço, pode-se treinar um agente que implemente a política ótima tendo como base sua experiência anterior.

O objetivo deste trabalho é verificar a aplicabilidade de uma solução baseada na aprendizagem por reforço para computação, de maneira online, do problema dos k-servos. Esta verificação será feita a partir da comparação do desempenho obtido pela solução proposta, em experimentos realizados com seqüências de requisições geradas aleatoriamente e de tamanhos variados, com os algoritmos Harmonic e Work Function citados anteriormente.

Ao agente são apresentadas situações ou percepções de seu ambiente (configurações dos servos, no caso do PKS), representadas por estados, aos quais o agente responde com ações (movimentos dos servos para atender um determinado nó demanda). Para cada ação tomada, uma avaliação de seu resultado, na forma de recompensa ou punição, é apresentada ao agente pelo ambiente. O processo de aprendizagem tem por finalidade orientar o agente a tomar as ações que venham a maximizar (minimizar) as recompensas (punições) recebidas. Deve-se levar em conta que uma ação tomada em um dado instante influencia não apenas a avaliação imediata, mas, também, sobre todas as outras ações que serão efetuadas a partir de então. Trata-se, portanto, do problema de como mapear estados do ambiente em ações do agente de forma a maximizar (minimizar) um dado retorno. 
Entretanto, à medida que aumenta o número de possíveis posições que os servos podem ocupar (número de nós) e o de servos, a dimensão da estrutura de armazenamento utilizada pela aprendizagem por reforço para se obter a política ótima necessária à solução do PKS também cresce, sendo esse crescimento de forma exponencial. Em razão disso, quando aplicada a problemas de maior porte, a abordagem aqui apresentada torna-se inviável do ponto de vista computacional. Esse não é um problema específico dos algoritmos baseados em aprendizagem por reforço, uma vez que outras estratégias também apresentam o mesmo problema, como, por exemplo, o algoritmo Work Function. Com o intuito de apresentar uma forma de contornar esse incoveniente, uma solução alternativa foi desenvolvida.

Esta solução alternativa é hierarquizada e utiliza a aprendizagem por reforço, aplicada a um número reduzido de possíveis estados e ações, e o método guloso, onde o critério de escolha do deslocamento dos servos é baseado na menor distância ao local de requisição.

Este texto está organizado da seguinte forma: no Capítulo 2, introduzemse noções gerais de computação online e análise competitiva, sendo estes conceitos fundamentais para a compreensão dos mecanismos de comparação entre algoritmos online. No Capítulo 3, apresentam-se aspectos básicos da aprendizagem por reforço e do algoritmo Q-Learning, de forma a possibilitar uma melhor compreensão da modelagem do problema. No Capítulo 4, é apresentado formalmente o problema dos $k$-servos e alguns algoritmos utilizados na sua solução, além de possíveis aplicações a outros problemas correlatos. No Capítulo 5, é apresentada a modelagem do PKS de acordo com a abordagem da aprendizagem por reforço. Ainda neste Capítulo, mostram-se os 
resultados obtidos nos experimentos realizados, além de uma comparação com dois algoritmos de solução do PKS citados na literatura. No Capítulo 6 é apresentado o algoritmo hierárquico de solução do PKS, que pode ser aplicado a problemas de maior porte, buscando minimizar a restrição do tamanho do problema, permitindo sua aplicação a um número maior de problemas. No Capítulo 7 são apresentadas as conclusões finais e as perspectivas de trabalhos futuros. 


\section{Capítulo 2}

\section{Computação Online e Análise Competitiva}

\subsection{Introdução}

De maneira geral, na computação online, um algoritmo deve decidir qual ação a ser tomada para uma entrada específica, sem informações sobre as entradas futuras. Por exemplo, como uma chamada telefônica deve ser roteada? Qual página deve ser removida da memória rápida quando uma requisição nova chega e todas as páginas da memória rápida estão ocupadas? Do ponto de vista formal, esse tipo de algoritmo tem sido bastante estudado na literatura, e aplicado a problemas de paginamento e acesso a memórias, acesso a listas, sistemas de tarefas métricas e outros. Na análise do comportamento dos algoritmos de solução, um aspecto bastante importante diz respeito ao seu desempenho. Dentro desse contexto, a qualidade de um algoritmo online é quantificada em relação ao melhor desempenho de um algoritmo que 
possui conhecimento completo das informações futuras. Por se tratar de um procedimento comparativo, atribui-se a ele o nome de análise competitiva, estabelecida entre o algoritmo em questão e o desempenho de um algoritmo ótimo offline (adversário), que possui o conhecimento completo das demandas futuras [5].

Neste capítulo, será vista a conceituação básica sobre computação online e análise competitiva, extraída sobretudo da referência [5]. Na Seção 2.2, conceitua-se um problema de otimização e sua classificação em relação ao conhecimento que se possui sobre a seqüência de requisições. Finaliza-se este capítulo com a Seção 2.3, apresentando os conceitos de competitividade e taxa de competitividade, importantes no estudo da análise de desempenho de algoritmos online.

\subsection{Problemas de Otimização Online e Offline}

Um problema de otimização pode estar associado tanto à maximização quanto à minimização de custos. Neste trabalho, o interesse e o desenvolvimento da teoria são baseados em problemas de minimização de custos. A extensão a problemas de maximização pode ser feita facilmente. Um problema de otimização $\mathcal{P}$ associado à minimização de custos consiste em um conjunto $\mathcal{I}$ de entradas (requisições, pedidos, demandas, estados), cujos elementos são representados por $I \in \mathcal{I}$, e uma função custo $\mathcal{C}$. Para cada entrada $I$, simboliza-se por $\mathcal{F}(I)$ o conjunto de soluções possíveis, ou seja, as saídas que podem ser produzidas por um dado algoritmo. Seja $O$ uma solução $(O \in \mathcal{F}(I))$, então pode-se associar um real positivo $\mathcal{C}(I, O)$, que 
representa o custo da saída $O$ associado à entrada $I$. O objetivo buscado é minimizar o valor de $\mathcal{C}(I, O)$.

Essa notação pode ser estendida para introduzir o papel associado a um algoritmo. Para tanto, seja $A L G$ um algoritmo de solução do problema $\mathcal{P}$ e seja $A L G(I)$ a saída produzida pelo algoritmo para uma dada entrada $I$, isto é, $A L G(I) \in \mathcal{F}(I)$. O custo associado pode ser representado por $\mathcal{C}(I, A L G(I))$. Um algoritmo ótimo de solução de $\mathcal{P}$ deve obedecer à equação, para qualquer entrada $I$ válida:

$$
O P T(I)=\min _{A L G(I) \in \mathcal{F}(I)} \mathcal{C}(I, A L G(I))
$$

ou seja, o custo associado ao algoritmo ótimo é inferior ou igual ao custo associado a qualquer outro algoritmo que produza soluções possíveis em $\mathcal{P}$. Observe que, para um mesmo algoritmo $A L G(I) \in \mathcal{F}(I)$, pode-se ter vários custos associados, dependendo da entrada $I$.

Os problemas de otimização podem ser classificados de várias formas, de acordo com o conhecimento que se possui sobre as entradas, as saídas e a função-custo. Problemas de otimização em variáveis contínuas ou discretas, estáticos ou dinâmicos, com ou sem restrições, com funções objetivo ${ }^{1}$ lineares ou não lineares, têm sido largamente estudados na literatura. Um aspecto particular que será abordado nesse trabalho, diz respeito à forma do conjunto de entradas $\mathcal{I}$. Um elemento $I$ desse conjunto corresponde a uma seqüência de demandas (ou solicitações de serviços), definida por:

$$
I \equiv \sigma=\left\{\sigma_{1}, \sigma_{2}, \ldots, \sigma_{i}, \ldots, \sigma_{m}\right\}
$$

\footnotetext{
${ }^{1}$ A função objetivo corresponde à função a ser otimizada. Neste trabalho, ela está representada pela função custo $\mathcal{C}(I, O)$, embora possa assumir outras formas.
} 
onde, para atender a cada demanda $\sigma_{i}$ um servo deve ser alocado. Deve-se observar que, para cada demanda, uma saída (ou estado ou nova configuração dos servos) é produzida e tem associada a ela um custo, proporcional ao deslocamento do servo.

A forma como essas demandas são apresentadas define duas classes de problemas de otimização. Se uma nova demanda só é conhecida após o processamento da anterior, tem-se um problema de otimização online. Mais precisamente, quando se está atendendo a demanda $\sigma_{t}, 1 \leq t \leq m$, não se tem nenhuma informação sobre a requisição $\sigma_{t^{\prime}}$, com $t^{\prime}>t$. A dificuldade inerente a este tipo de algoritmo é que cada saída online influencia o custo da solução global e a tomada de decisões se baseia somente em eventos passados. A outra classe é a de problemas offline onde, nesse caso, todas as demandas são conhecidas a priori.

Um bom exemplo de aplicações de problemas de otimização online é o algoritmo de paginação de memória de um sistema operacional (SO) ([8] e [19]). Considere um SO com um sistema de memória em dois níveis, consistindo de uma memória rápida e uma lenta. Aqui, cada pedido especifica uma página na memória do sistema. Um pedido é atendido se a página requisitada correspondente está na memória rápida. Se a página solicitada não está na memória rápida, uma falta de página ocorre. Neste instante, uma página deve ser removida da memória rápida para a lenta, de modo que a página requisitada possa ocupar este local vago na memória rápida. Um algoritmo de paginação deve especificar qual página da memória rápida deve ser desocupada, visando à minimização no número de faltas de páginas.

O principal impacto de processar entradas à medida que elas chegam é 
que, para qualquer algoritmo online, existirão entradas que forçarão o algoritmo a produzir respostas sub-ótimas. A questão que surge é: qual medida de desempenho seria apropriada para estudar e comparar o poder de algoritmos distintos? Sleator e Tarjan [2] sugeriram que a solução produzida por um algoritmo online para uma entrada específica deveria ser comparada com a solução obtida por um algoritmo ótimo offline para a mesma seqüência de entrada. Manasse [1] formalizou a idéia e introduziu a noção de competitividade.

\subsection{Análise Competitiva: Competitividade e Taxa de Competitividade}

Algoritmos online sob a ótica da análise competitiva têm sido, implícita e explicitamente, estudados há mais de 30 anos dentro do contexto de agendamento de tarefas, otimização, estrutura de dados e outros tópicos relacionados à computação [5]. De acordo com a análise competitiva, a qualidade de um algoritmo online para uma dada seqüência de entrada é quantificada pela sua comparação com o desempenho de um algoritmo ótimo offline, o qual possui conhecimento completo das demandas futuras.

Dado o algoritmo ótimo para a solução de $\mathcal{P}$ definido na Equação 2.1, diz-se que um algoritmo $A L G$ é uma c-aproximação assintótica de $O P T$ se

existe uma constante $\alpha \geq 0$ tal que, para toda entrada válida $I$, tem-se:

$$
A L G(I)-c \cdot O P T(I) \leq \alpha
$$

Se $\alpha=0$ diz-se que $A L G$ é uma c-aproximação de $O P T$. 
Por outro lado, diz-se que um algoritmo online $A L G$ é c-competitivo se existe uma constante $\alpha$ tal que, para toda entrada finita $I$, definida em 2.2, tem-se:

$$
A L G(I) \leq c \cdot O P T(I)+\alpha
$$

Se $\alpha \leq 0$ diz-se que $A L G$ é estritamente c-competitivo.

Diante dessas definições pode-se verificar que um algoritmo online $A L G$ (estritamente) c-competitivo é um algoritmo c-aproximado com a restrição adicional de que deve ser executado de forma online. O menor valor da constante $c$, para o qual $A L G^{2}$ é c-competitivo é chamada razão ou taxa de competitividade de $A L G$ e denotada por $\mathcal{R}(A L G)$.

A análise da competitividade de um dado algoritmo online pode ser estabelecida como um jogo entre um jogador online e um adversário particular. $\mathrm{O}$ jogador online tem seu comportamento determinado pelo algoritmo online associado, enquanto ao adversário é admitido o conhecimento completo da entrada do algoritmo, ou seja, seus movimentos podem ser estabelecidos através de um algoritmo offline ótimo. Além disso, a sequiência é normalmente escolhida pelo adversário como sendo uma daquelas mais desfavoráveis ao jogador online, como forma de maximizar a competitividade. Para algoritmos online determinísticos, o adversário conhece a priori todos os movimentos do jogador e pode determinar a sequiência maliciosa antecipadamente.

\footnotetext{
${ }^{2}$ Ressaltado que $A L G$ é determinístico. Um algoritmo determinístico é aquele cujo comportamento sempre produz a mesma saída para uma mesma seqüência de entrada (exemplo: algoritmo Work Function). Em oposição aos algoritmos determinísticos, existem os algoritmos randômicos cujas saídas podem variar para uma mesma seqüência de entrada. Normalmente, as decisões tomadas por este tipo de algoritmo são feitas a partir de probabilidades. (exemplo: algoritmo Harmonic).
} 
Para o caso de algoritmos online randômicos, a análise é um pouco mais sutil e não será abordada aqui.

$\mathrm{Na}$ análise propriamente dita, utiliza-se comumente o conceito de função potencial [29], definida em termos das configurações do algoritmo online e do seu adversário. Por configuração de um algoritmo, entende-se o estado do algoritmo com relação ao mundo exterior. Por exemplo, no caso de um algoritmo online de solução do PKS, uma configuração do mesmo corresponde a uma dada disposição dos servos [5]. Esse método é usado para estabelecer limites superiores de desempenho para algoritmos determinísticos e randômi$\cos$.

Um outro método de prova formal, baseado no teorema minimax de Yao [27], é denominado princípio minimax de Yao [8]. Dado um problema online, a taxa de competitividade do melhor algoritmo randômico online em relação ao ótimo offline é equivalente a taxa de competitividade do melhor algoritmo determinístico online para uma seqüência de entrada do pior-caso.

O estudo de métodos formais de análise de competitividade foge ao escopo do presente texto, não sendo, portanto, abordado neste trabalho. A introdução destes conceitos serve apenas para informar ao leitor dos métodos formais existentes. Informações adicionais podem ser obtidas nas referências supracitadas. 


\section{Capítulo 3}

\section{Problema dos $k$-Servos}

\subsection{Introdução}

O problema dos k-servos online foi introduzido por Manasse, McGeoch e Sleator [1]. Este modelo proposto provê uma abstração para um grande número de problemas. Mais ainda, a conjectura e o modelo dos k-servos têm proporcionado um significativo desenvolvimento da análise competitiva [5]. O problema dos $k$-servos é, provavelmente, o problema online mais influente $[22]$.

O problema dos k-servos é bem simples de ser descrito. Denominam-se servos as unidades de atendimentos das demandas ${ }^{1}$. Demanda é a indicação do local onde os atendimentos deverão ser realizados, ou seja, os servos devem atender às solicitações originadas nestes locais de demanda. Considere um espaço métrico $M$ habitado por $k$ servos localizados em pontos de $M$ não

\footnotetext{
${ }^{1}$ Os termos pedido por serviço, requisição e demanda são sinônimos e serão usados indistintamente.
} 
necessariamente distintos. Inicialmente, cada servo é posicionado em algum ponto de $M$. No decorrer do tempo, pedidos por serviço chegam de algum ponto do espaço métrico. A meta é servir a seqüência de pedidos. Um pedido j é simplesmente um ponto do espaço métrico, e servir $j$ implica em deslocar um servo ao ponto $j$. Associado ao deslocamento do servo localizado em $i$ para um local $j$, existe um custo de atendimento proporcional à distância $d(i, j)$ do servo em $i$ à demanda em $j$. O objetivo é minimizar a distância total percorrida pelos servos no atendimento de todas as requisições (vetordemanda). Portanto, um algoritmo que resolva o problema dos $k$-servos de maneira online deve decidir qual servo será deslocado para atender a cada uma das requisições, de modo que a distância total percorrida pelos mesmos seja a menor possível.

Inicia-se este capítulo, na Seção 3.2, introduzindo o conceito de espaço métrico e apresentando a definição formal do PKS. Na Seção 3.3, apresentamse algumas estratégias de solução do PKS, bem como as limitações inerentes a cada método, o que ocasiona a busca por soluções computacionais $k$ competitivas. Finaliza-se o capítulo, mostrando algumas aplicações do PKS e alguns de seus problemas correlatos.

\subsection{Conceitos Preliminares}

\subsubsection{Espaço Métrico}

Antes de abordamos a modelagem do problema dos $k$-servos, faz-se necessário conceituar espaço métrico [5]. Um espaço métrico $M$ é um par $(S, d)$, onde $S$ é um conjunto de nós e $d: S \times S \rightarrow R^{+}$é uma função custo de 
transição que satisfaz:

1. $d(i, j)>0, \forall i \neq j, i, j \in S$;

2. $d(i, i)=0, \forall i \in S$;

3. $d(i, k) \leq d(i, j)+d(j, k), \forall i, j, k \in S$; (Desigualdade Triangular)

4. $d(i, j)=d(j, i), \forall i, j \in S$.

Para compreender como um espaço métrico pode ser usado em problemas online abstratos, é só considerar $S$ como sendo um conjunto de pontos com todas as possíveis posições que um determinado servo pode ocupar, enquanto $d$ representa uma função custo de transição entre pontos de $S$.

\subsubsection{Definição Formal do PKS}

Considere o grafo $G=\{X, U\}$, onde $X \subset S$ é o conjunto de nós de $G$, $\operatorname{com}|X|=n ; U(U=\{a \mid a=(i, j), i$ e $j \in X\})$ é o conjunto de arestas de $G$ e $d(i, j)$ é uma ponderação associada a aresta que liga o nó $i$ ao nó $j$. O algoritmo que resolve o problema dos k-servos provê o agendamento dos movimentos dos $k$ servos localizados em pontos do grafo $G$. Ao algoritmo, é apresentada uma seqüência de pedidos $\sigma=\left\{\sigma_{1}, \sigma_{2}, \ldots, \sigma_{m}\right\}$, onde cada pedido representa um ponto no grafo. Ao movimentar os servos, de $i$ para um local $j$, existe um custo de atendimento proporcional à distância percorrida $d(i, j)$. O algoritmo deve atender a todos os pedidos seqüencialmente. O objetivo em questão é atender a todas as requisições, minimizando a distância

total, $\sum_{l=1}^{m} d_{l}(i, j)$, percorrida pelos servos. 


\subsection{Estratégias de Solução}

O problema dos k-servos apresenta vários algoritmos capazes de solucionálo. Porém, diante da existência de uma solução online $k$-competitiva [1], a busca por novas soluções ainda não cessou, já que, até o presente momento, ninguém conseguiu provar que as soluções já encontradas são k-competitivas para todos os casos. Nesta Seção, são apresentadas algumas das soluções encontradas para o PKS, partindo inicialmente de soluções bastante simplórias, como é o caso do algoritmo proposto por Sleator e Tarjan e da estratégia gulosa, até chegar-se em soluções mais sofisticadas, cujas competitividades já foram provadas pelos pesquisadores ([22] e [23]), como é o caso dos algoritmos Harmonic e Work Function.

\subsubsection{Algoritmos Heurísticos}

\section{Algoritmo de Sleator e Tarjan para o Caso Online}

Sleator e Tarjan [1] apresentam um algoritmo para a solução dos k-servos online. A estratégia é, basicamente, escolher os movimentos que permitam que os diversos servos se desloquem de forma igualitária. O algoritmo mantém a distância total percorrida por cada servo $k$ na variável $D_{k}$. Se existe uma demanda $\sigma_{i}$ a ser atendida, o servo $k$ a ser escolhido para fazer o atendimento será aquele que minimize a expressão $D_{k}+d\left(j_{k}, \sigma_{i}\right)$, onde $d\left(j_{k}, \sigma_{i}\right)$ é a distância percorrida por um servo $k$ localizado em um nó $j$ até a requisição $\sigma_{i}$

O algoritmo de Sleator e Tarjan é apresentado na Figura 3.1. De acordo com o algoritmo, $G=\{X, U\}$ é um grafo ponderado, onde $X \subset M$ é o 
conjunto de nós de $G$, com $|X|=n ; U(U=\{a \mid a=(i, j), i$ e $j \in X\})$ é o conjunto de arestas de $G$ e $d(i, j)$ é uma ponderação associada a aresta que liga o nó $i$ ao nó $j$ do grafo $G$; $D$ é um vetor de tamanho $k$, contendo a distância acumulada percorrida pelos $k$ servos; $S$ é um conjunto de $k$ servos; $\sigma=\left\{\sigma_{1}, \sigma_{2}, \ldots, \sigma_{m}\right\}$ um vetor contendo uma seqüência de requisições $\sigma_{i} ; j_{k}$ é o nó onde está localizado o servo k e Sol é um vetor de tamanho $m$ que armazena o conjunto de soluções encontradas, ou seja, na posição $i$ deste vetor contém o número do nó em que estava localizado o servo que atendeu à demanda $\sigma_{i}$.

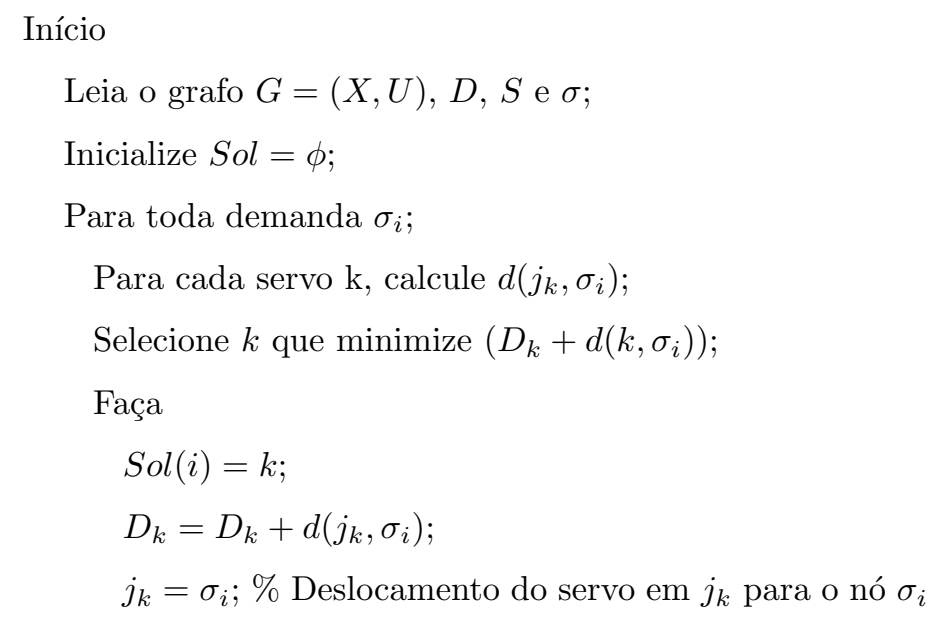

Fim

Fim

Fim

Figura 3.1: Algoritmo de Sleator e Tarjan.

Examinando este algoritmo, fica evidente que a estratégia proposta não é capaz de garantir a minimização do deslocamento dos servos para todos os 
casos, podendo esta afirmação ser evidenciada no exemplo a seguir. Considere o grafo abaixo (Figura 3.2), com os servos $s_{1}$ e $s_{2}$ ocupando, respectivamente, as posições 3 e 5 do grafo. O vetor demanda a ser atendido é [ 6 2] .
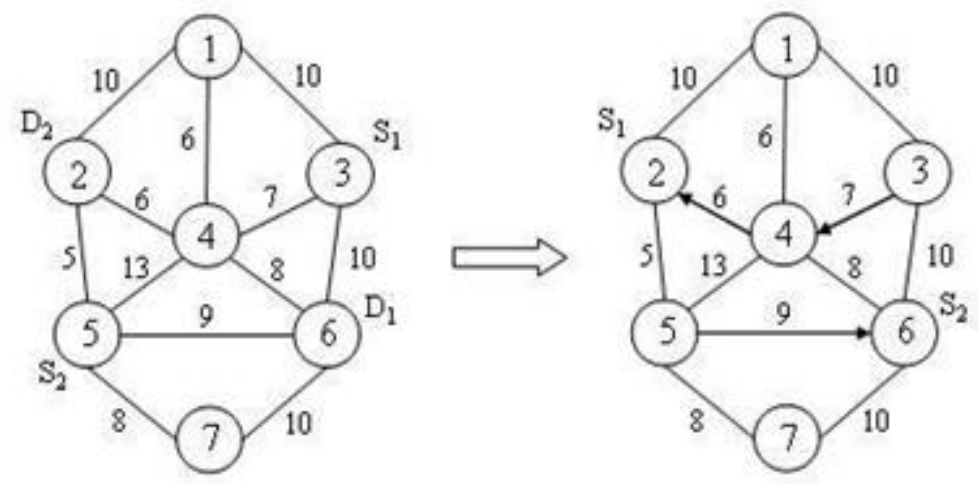

Figura 3.2: Limitação do algoritmo online de Sleator e Tarjan.

Pela análise do algoritmo, vê-se que a primeira demanda será atendida pelo servo $s_{2}$ e a segunda por $s_{1}$, sendo a distância total percorrida pelos mesmos igual a 22. Se for trocada a ordem do atendimento, $\operatorname{com} s_{1}$ atendendo à demanda em 6 e $s_{2}$ atendendo à demanda em 2 , obter-se-ia um custo total de 15, evidenciando que o algoritmo de Sleator e Tarjan não é capaz de garantir uma solução ótima para todos os casos.

\section{Algoritmo Guloso}

Para o problema dos k-servos, define-se um algoritmo online guloso [21] como aquele que processa cada pedido no intuito de minimizar o custo individual de servi-lo . Em outras palavras, o servo que atenderá ao pedido será aquele que estiver mais próximo da requisição. A figura 3.3 apresenta o algoritmo guloso em português estruturado. 
Início

Para toda demanda $\sigma_{i}$;

Desloque o servo cuja distância ao nó de demanda $\sigma_{i}$ seja a menor possível;

Fim

Fim

Figura 3.3: Algoritmo Guloso.

É facil perceber porque a estratégia gulosa não é necessariamente competitiva. Considere o grafo da Figura 3.4 e um problema com dois servos. Nesta Figura, $d(a, b)<d(b, c)$. Independente de qual for a posição inicial dos dois servos, a estratégia gulosa deve atender a seqüência de pedidos $c, b, a, b, a, \ldots$ da seguinte maneira: um servo permanecerá no nó $c$ para sempre, enquanto o outro atenderá aos pedidos em $a$ e $b$ e incorrerá em um custo ilimitado. Claramente, o custo ótimo offline para atender a esta sequiência de requisições será $d(a, b)+2 d(b, c)$, independentemente da configuração inicial dos servos.

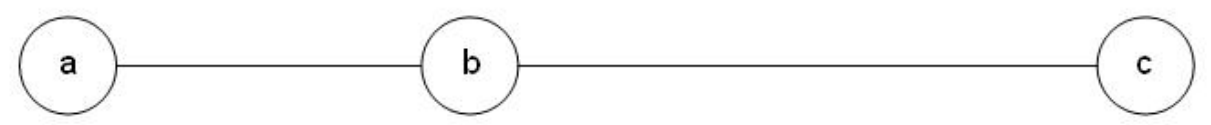

Figura 3.4: A estratégia gulosa não garante solução ótima. 


\subsubsection{Algoritmo Harmonic}

O Algoritmo Harmonic ([5] e [23]) para solução do Problema dos k-Servos foi proposto por Raghavan e Snir [4], e opera da seguinte maneira: a escolha do servo que atenderá a requisição $\sigma$ é feita de maneira probabilística, onde a cada servo é associada uma probabilidade cujo valor é inversamente proporcional à sua distância ao nó requisitado. Mais precisamente, considere $S=\left\{s_{1}, s_{2}, \ldots, s_{k}\right\}$ um conjunto de servos online e suponha que nenhum deles esteja localizado no nó de demanda corrente. Para cada $1 \leq i \leq k$, tem-se $d_{i}=d\left(s_{i}, \sigma\right)$, que representa a distância do servo $s_{i}$ à demanda $\sigma$. O Harmonic serve ao pedido $\sigma$, com o servo $s_{i}$ escolhido com probabilidade $\operatorname{Pr}\left(s_{i}, \sigma\right)=\frac{1}{N \cdot d\left(s_{i}, \sigma\right)}$, onde $N=\sum_{i=1}^{k} \frac{1}{d\left(s_{i}, \sigma\right)}$ é o fator de normalização. Portanto, um servo localizado em $i$ é escolhido randomicamente para atender a uma demanda em $\sigma$ de acordo com a probabilidade $\operatorname{Pr}\left(s_{i}, \sigma\right)$, de tal forma que quanto maior o valor de $\operatorname{Pr}\left(s_{i}, \sigma\right)$ maior a chance do servo $s_{i}$ ser escolhido para atender $\sigma$.

O algoritmo Harmonic pode ser visto como uma versão randômica de um algoritmo guloso determinístico, onde cada servo é escolhido de acordo com sua proximidade em relação à demanda, ou seja, o servo a ser escolhido será aquele cuja distância em relação ao nó requisitado for a menor dentre todos os servos.

O algoritmo Harmonic em português estruturado é apresentado na Figura 3.5 . 
Início

Para cada servo $k$, calcular $d\left(s_{i}, \sigma\right)$;

Encontre o fator de normalização $N=\sum_{i=1}^{k} \frac{1}{d\left(s_{i}, \sigma\right)}$;

Para cada servo $k$, calcule $\operatorname{Pr}\left(s_{i}, \sigma\right)=\frac{1}{N \cdot d\left(s_{i}, \sigma\right)}$;

Escolha randomicamente o servo $s_{i}$ a ser deslocado para atender $\sigma$ de acordo com as probabilidades calculada no passo anterior;

$\sigma=s_{i} ; \%$ Deslocamento de $s_{i}$ até $\sigma$

Fim

Figura 3.5: Algoritmo Harmonic.

Para facilitar a compreensão do funcionamento do algoritmo Harmonic, considere o grafo da Figura 3.6. Este grafo apresenta três servos, $\left[\begin{array}{lll}s_{1} & s_{2} & s_{3}\end{array}\right]$, que irão atender a uma demanda localizada em $\sigma$. A distância de cada servo à demanda é igual a $d\left(s_{1}, \sigma\right)=10, d\left(s_{2}, \sigma\right)=20$ e $d\left(s_{3}, \sigma\right)=30$.

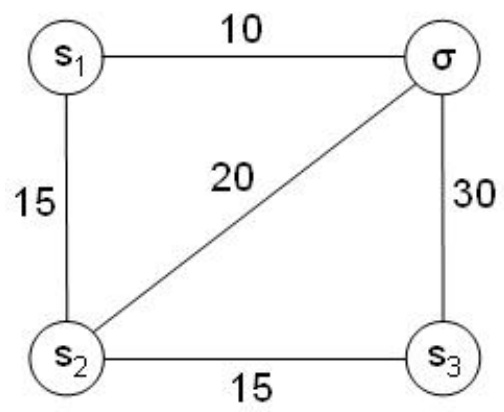

Figura 3.6: Funcionamento do algoritmo Harmonic.

O fator de normalização $N$, calculado de acordo com a expresão mostrada 
acima, é igual a:

$$
N=\frac{1}{10}+\frac{1}{20}+\frac{1}{30}=\frac{6+3+2}{60}=11 / 60=0.184
$$

Considere o símbolo $\operatorname{Pr}\left(s_{i}, \sigma\right)$ como a probabilidade do servo $s_{i}$ atender ao nó de demanda $\sigma$. Desta maneira, a probabilidade dos servos $\left[\begin{array}{lll}s_{1} & s_{2} & s_{3}\end{array}\right]$ serem escolhidos é dado pela expressão $\frac{1}{N \cdot d\left(s_{i}, \sigma\right)}$. Assim, tem-se:

- $\operatorname{Pr}\left(s_{1}, \sigma\right)=\frac{1}{N d\left(s_{1}, \sigma\right)}=\frac{1}{0.184 \cdot 10}=\frac{1}{1.84}=0.54$

- $\operatorname{Pr}\left(s_{2}, \sigma\right)=\frac{1}{N d\left(s_{2}, \sigma\right)}=\frac{1}{0.184 \cdot 20}=\frac{1}{3.68}=0.27$

- $\operatorname{Pr}\left(s_{3}, \sigma\right)=\frac{1}{N d\left(s_{3}, \sigma\right)}=\frac{1}{0.184 \cdot 30}=\frac{1}{5.52}=0.19$

Os valores de $\operatorname{Pr}$ representam as chances que cada servo possui de ser escolhido para atender a demanda $\sigma$. Deste modo, estes valores indicam que $s_{1}$ tem $54 \%, s_{2}$ tem $27 \%$ e $s_{3}$ tem $19 \%$ de chance de, respectivamente, serem escolhidos para atender à demanda $\sigma$. Durante a implementação, deve-se garantir que a escolha randômica dos deslocamentos dos servos seja feita em função de sua probabilidade $\operatorname{Pr}$ de escolha, de tal forma que servos com menores probabilidades também possam ser escolhidos.

\subsubsection{Algoritmo Work Function (WFA) - Função de Tra- balho}

O Algoritmo Work Function ([5] e [22]) é relativamente simples de ser implementado, porém, sua descrição formal é bastante complexa e, conseqüentemente, difícil de ser entendida. Portanto, neste texto, abordar-se-ão 
os aspectos da implementação do algoritmo, comentando, somente se indispensável, os aspectos formais relacionados.

O WFA desempenha um importante papel na análise competitiva de algoritmos online determinísticos [5], já que o funcionamento do algoritmo Work Function é baseado no registro dos custos offline ótimos da distância total percorrida até a demanda anterior à corrente.

A aplicação do algoritmo Work Function não se restringe somente ao problema dos k-servos. A aplicação deste tipo de algoritmo pode ser feita a uma ampla gama de problemas. Em seu trabalho, Koutsoupias [30] provou que a taxa de competitividade do Work Function é no máximo $2 k-1$. Este fato faz com que este algoritmo seja bastante aplicado e estudado dentro do problema dos $k$-servos.

Antes de iniciar a explicação do funcionamento do WFA, alguns conceitos preliminares são necessários. Dado um grafo $G=\{X, U\}$, onde $X \subset M$ é o conjunto de nós de $G$, com $|X|=n ; U(U=\{a \mid a=(i, j), i$ e $j \in X\})$ é o conjunto de arestas de $G$ e $d$ é uma ponderação associada a cada aresta, uma configuração de qualquer algoritmo que resolva o problema dos $k$-servos é vista como um conjunto de $k$ pontos, não necessariamente distintos, representando a localização dos servos em $G$. Deve-se ter em mente que uma configuração é um conjunto com, no mínimo, um elemento, e que dois servos podem ocupar o mesmo nó em um dado instante. Denota-se por letras maiúsculas a configuração dos servos e pontos de $G$ por letras minúsculas. 
Para configurações $C_{1}$ e $C_{2}$, considere $C_{1}+C_{2}$ a união entre conjuntos ${ }^{2}$; similarmente, $C_{1}-C_{2}$ representa a diferença ${ }^{3}$. Como exemplo, considere uma configuração $C_{1}=\{a b c\}$. O resultado de $C_{1}-\{a\}+\{d\}$ é uma nova configuração, $C_{2}$, que será obtida excluindo-se o elemento $a$ e adicionando-se o elemento $d$ a $C_{1}$, resultando em $C_{2}=\{b c d\}$. Para quaisquer configurações $C_{1}$ e $C_{2}$, define-se $D\left(C_{1}, C_{2}\right)$, a distância entre configurações, como o valor mínimo do somatório da distância entre pontos distintos entre as configurações $C_{1}$ e $C_{2}$. Por exemplo, se a distância entre os pontos $a$ e $b$ for 1 , $D(\{a c d\},\{b c d\})=1$, já que os pontos distintos entre as configurações $C_{1}$ e $C_{2}$ são $a$ e $b$. O valor de $D(\{a c d\},\{b c d\})=1$, representa o custo de se deslocar um servo do nó $a$ para o $b$.

$\mathrm{O}$ algoritmo Work Function registra os valores a serem processados em uma tabela. Os valores são computados de maneira recursiva, utilizando-se programação dinâmica, de tal forma que o valor na linha $i+1$ da tabela é calculado tomando-se como base os valores obtidos na linha $i$ anterior.

A primeira etapa do algoritmo Work Function é calcular o número de configurações possíveis dos servos. Este valor é dado pela expressão $\mathcal{C}_{n, k}=$ $\frac{n !}{(n-k) ! k !}$, onde $n$ é o tamanho do grafo $G$ e $k$ o número de servos. Este valor representa o número total de colunas da tabela. Infere-se, portanto, que cada coluna da tabela representará uma configuração possível dos servos. O

\footnotetext{
${ }^{2}$ Dado dois conjuntos $C_{1}$ e $C_{2}$, a união entre conjuntos $C_{1}+C_{2}$ resulta em um conjunto $C$ cujos elementos $x$ são calculados de acordo com a expressão $C=C_{1}+C_{2}=\{x \mid x \in$ $C_{1}$ ou $\left.x \in C_{2}\right\}$

${ }^{3}$ Dado dois conjuntos $C_{1}$ e $C_{2}$, a diferença entre conjuntos $C_{1}-C_{2}$ resulta em um conjunto $C$ cujos elementos $x$ são calculados de acordo com a expressão $C=C_{1}-C_{2}=$ $\left\{x \mid x \in C_{1}\right.$ e $\left.x \notin C_{2}\right\}$
} 
espaço de memória necessário para armazenar a tabela será, portanto, $\mathcal{C}_{n, k} \cdot m$, onde $m$ é o número total de demandas. Logo, a complexidade em espaço do algoritmo é $\mathcal{O}\left(m \cdot n^{k}\right)$.

Considere uma seqüência de solicitações $\sigma=\left\{\sigma_{1}, \sigma_{2}, \ldots, \sigma_{m}\right\}$, o número de linhas da tabela será igual ao tamanho do vetor $\sigma$ mais um, matematicamente, $|\sigma|+1$. A razão do número de linhas ser igual a $|\sigma|+1$ se deve ao fato de na primeira linha da tabela ser armazenado o valor de todas as distâncias entre configurações de uma configuração inicial $C_{0}$ e uma configuração $C$ válida. Matematicamente, na primeira linha teremos $D\left(C, C_{0}\right)$. Nesta linha, considera-se o vetor de requisições vazio, sendo representado pelo símbolo $\phi$. As demais linhas, representam o cômputo do algoritmo para cada requisição $\sigma_{i}$ em todas as configurações $C$ válidas.

Para facilitar a compreensão, considere o grafo da Figura 3.7. Considere, ainda, como configuração inicial dois servos localizados em [1 2], ou seja, $C_{0}=\left[\begin{array}{ll}1 & 2\end{array}\right]$, e uma demanda a ser atendida no nó $4, \sigma=4$. O número de colunas da tabela é igual a $\mathcal{C}_{4,2}=6$ e o número de linhas é igual a 2 . Na primeira linha da tabela (Ver Tabela 3.1), tem-se a distância entre $C_{0}$ e todas as 6 configurações possíveis de servos. Por exemplo, a distância entre $C_{0}=\left[\begin{array}{ll}1 & 2\end{array}\right]$ e $C=\left[\begin{array}{ll}3 & 4\end{array}\right]$ é 2 , indicando o valor mínimo do somatório da distância entre as duas configurações ${ }^{4}$. Formalmente, $D\left(C_{0}, C\right)=2$. Os demais valores da linha 1 podem ser calculados similarmente.

Os valores da linha 2, são calculados tomando-se como base os valores

\footnotetext{
${ }^{4}$ Este valor representa o custo de se deslocar um servo do nó 1 ao 4, e outro servo do nó 2 ao 3. Observe que se fosse deslocado o servo do nó 1 ao 3 , e o outro servo do nó 2 ao 4 , o valor de $D\left(C_{0}, C\right)$ seria 4 . Desta forma, o valor mínimo entre as configurações é 2 , por ser o menor valor obtido possível.
} 


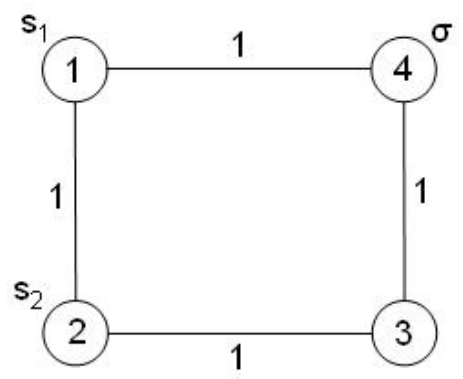

Figura 3.7: Grafo.

obtidos na linha 1 através da expressão:

$$
w_{i}(C)=\min _{x \in C}\left\{w_{i-1}\left(C-x+\sigma_{i}\right)+d\left(x, \sigma_{i}\right)\right\}
$$

Esta expressão calcula o valor da função de trabalho da linha $i$, para $i>1$, para uma dada configuração $C$. Observe que se a demanda está localizada em um mesmo ponto que um dos servos da configuração $C$, o valor da função de trabalho da linha $i$ será igual ao valor da $(i-1)$-ésima linha, indicando que nenhum deslocamento precisa ser efetuado. O significado de cada um dos termos desta expressão é o seguinte:

- $d\left(x, \sigma_{i}\right)$ : distância entre o servo $x$ e a demanda $\sigma_{i}$.

- $w_{i-1}\left(C-x+\sigma_{i}\right)$ : valor da função de trabalho armazenado na coluna referente à configuração $C-x+\sigma_{i}$ da linha $i-1$, onde $C$ é uma configuração válida e $x$ é um dos servos da configuração $C$ e $\sigma_{i}$ é a demanda corrente;

- $\min _{x \in C}$ : o valor de $w_{i}(C)$ será igual ao menor valor obtido pela expressão $w_{i-1}\left(C-x+\sigma_{i}\right)+d\left(x, \sigma_{i}\right)$, onde $x$ é um dos servos da configuração $C$ e $\sigma_{i}$ é a demanda corrente; 
Como exemplo, considere o cálculo do valor da configuração $C=\left[\begin{array}{ll}1 & 2\end{array}\right]$ (coluna 1) da primeira demanda (linha 2$)^{5}$. Aqui, o conjunto de servos é $x=$ [1 2] (número de servos igual a 2) e a demanda corrente é $\sigma_{i}=4$. Os servos serão escolhidos de acordo com os valores obtidos no cálculo da sua função de trabalho $w_{i}(C)=w_{i-1}\left(C-x+\sigma_{i}\right)+d\left(x, \sigma_{i}\right)$. Desta maneira, para cada entrada da tabela, dois valores de função de trabalho $w$, uma para cada servo, serão calculados. O servo que obtiver o menor entre os dois valores da função de trabalho será denotado por $x_{\min }=\arg \min _{x \in C}\left\{w\left(C-x+\sigma_{i}\right)+d\left(x, \sigma_{i}\right)\right\}$, e o valor da sua função trabalho respectiva será armazenado na entrada correspondente da tabela. O outro valor será desprezado. Assim, o servo $x_{\text {min }}$ será o escolhido para atender à demanda $\sigma_{i}$, e o custo associado será igual a $d\left(x_{\min }, \sigma_{i}\right)$, a distância entre o servo e a demanda.

- $w_{2}\left(\left[\begin{array}{ll}1 & 2\end{array}\right]\right)=\min _{x \in\{1,2\}}\left\{\left[\left(w_{1}\left(\left[\begin{array}{ll}1 & 2\end{array}\right]\right)-\mathbf{1}+4\right)+d(1,4)\right],\left[\left(w_{1}\left(\left[\begin{array}{ll}1 & 2\end{array}\right]\right)-\mathbf{2}+\right.\right.\right.$ 4) $+d(2,4)]\}$

- $w_{2}\left(\left[\begin{array}{ll}1 & 2\end{array}\right]\right)=\min \left\{\left[w_{1}([24])+1\right],\left[w_{1}\left(\left[\begin{array}{ll}1 & 4\end{array}\right]\right)+2\right]\right\}=2$

Voltando ao exemplo, partindo-se da configuração $C_{0}=\left[\begin{array}{ll}1 & 2\end{array}\right]$, o servo que irá atender à demanda $\sigma_{i}=4$ será aquele que obtiver menor função de trabalho, e o custo correspondente será igual a distância percorrida por ele para atender à demanda. Assim, neste exemplo, $x_{\min }=1$, o servo 1 irá atender à demanda em 4 e o custo correspondente será igual a $d(1,4)=1$. Os demais valores da linha 2 são calculados similarmente.

\footnotetext{
${ }^{5} \mathrm{O}$ valor armazenado correspondente a uma determinada linha e coluna de uma tabela é denominado de entrada.
} 
Tabela 3.1: Exemplo de aplicação do algoritmo Work Function.

\begin{tabular}{|c|c|c|c|c|c|c|}
\hline & \multicolumn{7}{|c|}{ Configurações dos servos } \\
\hline Demanda & {$\left[\begin{array}{ll}1 & 2\end{array}\right]$} & {$\left[\begin{array}{ll}1 & 3\end{array}\right]$} & {$\left[\begin{array}{ll}1 & 4\end{array}\right]$} & {$\left[\begin{array}{ll}2 & 3\end{array}\right]$} & {$\left[\begin{array}{ll}2 & 4\end{array}\right]$} & {$\left[\begin{array}{ll}3 & 4\end{array}\right]$} \\
\hline$\phi$ & 0 & 1 & 2 & 2 & 1 & 2 \\
\hline 4 & 2 & 3 & 2 & 2 & 1 & 2 \\
\hline
\end{tabular}

Deve-se inferir que os valores armazenados na tabela, que se referem aos valores da função de trabalho $w$ calculados, somente são utilizados para a escolha do deslocamento dos servos, devendo ser deslocado o servo que apresentar o menor valor de $w$ para cada configuração e demanda correspondentes. $\mathrm{O}$ custo associado não está relacionado aos valores armazenados na tabela, e sim, ao custo do deslocamento entre o servo escolhido e o nó de demanda.

O algoritmo Work function em Português Estruturado é apresentado na Figura 3.8.

Deve ser observado que, a partir de uma configuração inicial $\mathcal{C}_{i-1}$ (a qual é representada por uma das colunas da tabela) e quando ocorre uma requisição $\sigma_{i}$, escolhe-se, utilizando os valores calculados da função de trabalho $w_{i}(C)$, qual o servo $\left(x_{\min }\right)$ a ser deslocado e registra-se a distância $\left(d\left(x_{\min }, \sigma_{i}\right)\right)$ percorrida pelo mesmo até o nó de demanda. Em seguida, uma nova configuração $C_{i}$ será alcançada, sendo a mesma representada por uma outra coluna da tabela ${ }^{6}$. Neste momento, atualiza-se os pontos da configuração atual, que passará a ser $C_{i}=C_{i-1}-\sigma_{i}+x_{\min }$, e a coluna da tabela que corresponde a esta configuração (col_atual). Novamente, os valores de $w_{i}(C)$ para esta

\footnotetext{
${ }^{6} \mathrm{~A}$ configuração $C_{i}$ será igual a $\mathcal{C}_{i-1}$ quando o nó de demanda estiver localizado em um mesmo ponto que um dos servos da configuração $\mathcal{C}_{i-1}$.
} 
Início

Encontre o conjunto de configurações $C$ válidas dos servos;

Encontre os valores de $D\left(C, C_{0}\right)$ e armazene em cada configuração $C$

respectiva na primeira linha da tabela;

Observe a coluna $\operatorname{col}_{i-1}$ da tabela que corresponde à configuração $C_{0}$;

$C_{i}=C_{0} ; \%$ coluna atual da tabela - estado atual dos servos

$\mathcal{C}_{i-1}=C_{0} ; \%$ coluna anterior da tabela - estado anterior da tabela

Para toda demanda $\sigma_{i}$

Para cada configuração $C$ (coluna da tabela)

Encontre o valor de $w_{i}(C)=\min _{x \in C}\left\{w_{i-1}\left(C-x+\sigma_{i}\right)+d\left(x, \sigma_{i}\right)\right\}$;

Encontre o servo $x_{\text {min }}=\arg \min _{x \in C}\left\{w\left(C-x+\sigma_{i}\right)+d\left(x, \sigma_{i}\right)\right\}$;

Armazene o menor valor de $w$ na entrada correspondente da tabela e o

servo $x_{\min }$ a ser delocado;

Fim

Partindo-se da coluna $\operatorname{col}_{i}$, observe o servo $x_{\min }$ e registre o deslocamento

$d\left(x_{\min }, \sigma_{i}\right)$ até o servo $\sigma_{i} ;$

$\sigma_{i}=x_{\min }$

$\mathcal{C}_{i-1}=C_{i}$

Observe a nova configuração $C_{i}=C_{i-1}-\sigma_{i}+x_{\min }$ e a coluna $\operatorname{col}_{i}$ da tabela que corresponde a esta configuração;

Fim

Fim

Figura 3.8: Algoritmo Work Function.

nova configuração serão utilizados para a escolha do servo a ser deslocado e a distância percorrida pelo mesmo até a demanda será registrada. O procedimento para a escolha do agendamento dos servos irá se repetir até que a seqüência de requisições seja totalmente atendida. 


\subsection{Aplicações}

O problema dos k-servos serve como uma abstração para um grande número de problemas. A seguir, apresentam-se alguns exemplos de problemas que podem ser visualizados de acordo com a modelagem do PKS.

- Problema de paginação ([8] e [19]): uma instância do PKS com um espaço métrico $M=(S, d)$ uniforme (todas as distâncias são iguais a 1), onde $k$ servos representam páginas da memória rápida e $|M|=n$ é o número de páginas na memória lenta;

- Disco de leitura/escrita ([12] e [13]): Duas cabeças de leitura/escrita se movem em um segmento de linha que é o raio do disco. À medida que o disco gira, elas podem acessar qualquer registro do disco. Um algoritmo deve determinar qual das duas cabeças deve se deslocar no intuito de servir ao pedido de leitura/escrita em uma dada posição do disco. Uma maneira de quantificar o desempenho do sistema é medir a distância total percorrida pelas cabeças. Este problema é equivalente ao problema de 2 servos em um segmento de linha.

- k-servos com janela de tempo [14]: em situações reais de atendimento de múltiplas demandas com múltiplos servos, um dos fatores mais importantes para o desempenho é o tempo de atendimento. Quando esse fator é preponderante, obviamente, ele será considerado como o custo de deslocamento. Em outras situações, entretanto, sua consideração será tipicamente restritiva. Dessa forma, em sistemas de atendimento de emergência, é necessário considerar a restrição de tempo. 


\section{Problemas Correlatos ao PKS}

Os problemas correlatos segue o mesmo modelo padrão do PKS, sendo adicionadas, entretanto, algumas restrições especiais. Trata-se, portanto, de uma variação do modelo padrão do problema dos k-servos. Abaixo, são apresentados alguns problemas correlatos ao PKS.

- Problema de Múltiplos Servos: Batta e Mannur [15] estudam situações de atendimento de emergência com vários tipos de servos em que um atendimento exige a presença de um servo de cada tipo no ponto de demanda;

- Modelos hierárquicos para atendimento dinâmico ([16], [17] e [18]): eventualmente, os clientes demandantes podem ser classificados de forma que as exigências para o atendimento possam ser relaxadas para certos subconjuntos de usuários. 


\section{Capítulo 4}

\section{Aprendizagem por Reforço}

\subsection{Introdução}

A aprendizagem por reforço (do inglês, reinforcement learning) é um tema multidisciplinar, envolvendo disciplinas como Biologia, Ciências da Computação, Ciências Cognitivas, Engenharia, Filosofia, Física, Matemática, Psi-

cologia e Sociologia. Trata-se de um ramo do aprendizado de máquinas ${ }^{1}$ que estuda o aprendizado a partir da interação entre agente e seu ambiente. Um agente aprendiz é aquele que, a partir da interação com o ambiente que o cerca, aprende de maneira autônoma uma política ótima de atuação por experimentação direta, sem ser ensinado por meio de exemplos fornecidos por um supervisor. O ambiente é todo o sistema físico externo que irá interagir com o agente, percebido por este através de um conjunto de informações denominado de estado do ambiente.

\footnotetext{
${ }^{1}$ Campo da inteligência artificial cujo interesse é a construção de programas de computadores que se aperfeiçoam automaticamente com a experiência.
} 
De uma maneira informal, a aprendizagem por reforço é uma forma de aprendizado não-supervisionado ${ }^{2}$ que ensina como mapear estados para ações, de modo a maximizar (minimizar) um sinal numérico de retorno, que representa a soma total de todas as recompensas (punições) decorrentes das ações tomadas. Não são ditas ao agente de aprendizagem quais ações devem ser tomadas, assim como ocorre em outras abordagens. O agente deve descobrir quais ações resultam nas maiores recompensas (menores punições) a partir das percepções do ambiente, representadas por estados, que lhe são apresentadas. Para cada ação tomada, uma avaliação do seu resultado, na forma de recompensa ou punição, é apresentada ao agente pelo ambiente. O processo de aprendizagem tem por finalidade orientar o agente a tomar as ações que venham a maximizar (minimizar) as recompensas (punições) recebidas.

O agente indica quais as melhores ações a serem tomadas em um estado específico, atribuindo a cada uma delas uma probabilidade de ser escolhida, sendo, obviamente, associado uma probabilidade mais elevada de ser escolhida às ações que proporcionam um maior (menor) retorno total. A este mapeamento de estados em ações denomina-se política. Se esta política produzir um retorno total máximo (mínimo), dizemos que ela é ótima.

A aprendizagem por reforço possibilita o aprendizado diretamente a partir da interação com o ambiente, sem ter que considerar qualquer situação que não seja realmente experimentada e sem ter qualquer habilidade para predizer como o ambiente deve se comportar em resposta às suas ações.

\footnotetext{
${ }^{2}$ Aprendizado em que não é necessário um supervisor indicando a resposta (saída) desejada para uma dada entrada específica.
} 
Existem grandes diferenças entre o método de aprendizado utilizado pela aprendizagem por reforço e aqueles baseados em aprendizado supervisionado. A diferença mais importante está na inexistência de um supervisor que informa ao agente o quão bem ele está atuando. No aprendizado supervisionado, usualmente, é apresentado ao agente um conjunto de treinamento, com os valores de entradas (o estado do ambiente e a ação do agente) e os resultados esperados. Com base na diferença entre os resultados obtidos e os esperados, o agente pode verificar e corrigir o seu funcionamento. Neste método de aprendizagem, existe um agente externo que fornece os pares de dados ao sistema e verifica a corretude da saída obtida.

Se o aprendizado é baseado em reforço, a avaliação da atuação do agente acontece simultaneamente ao aprendizado. A interação com o ambiente acontece continuamente. O agente seleciona ações; o ambiente responde a estas ações e apresenta novas situações ao agente. No modelo de aprendizagem por reforço utilizado neste trabalho, as ações são realizadas sobre uma seqüência de instantes de tempo discretos $t_{i}(i=1,2, \ldots, m)$, de tal forma que a cada instante de tempo, o agente observa o estado $s_{t}$ do ambiente e seleciona uma ação específica. A resposta do ambiente ocorre na forma de um sinal de reforço, sendo o mesmo um valor numérico. Tomando como base os sinais de reforço recebidos ao longo do tempo, e ao mesmo tempo que visita estados e executa ações, o agente realiza seu aprendizado[6].

A aprendizagem por reforço é, antes de tudo, indicada quando se deseja obter a política ótima nos casos em que não se conhece a priori a função que modela esta política. O agente deve interagir com seu ambiente diretamente para obter informações que serão utilizadas para a obtenção da política ótima. 
Um diagrama esquemático de um sistema de aprendizagem por reforço é apresentado na Figura 4.1.

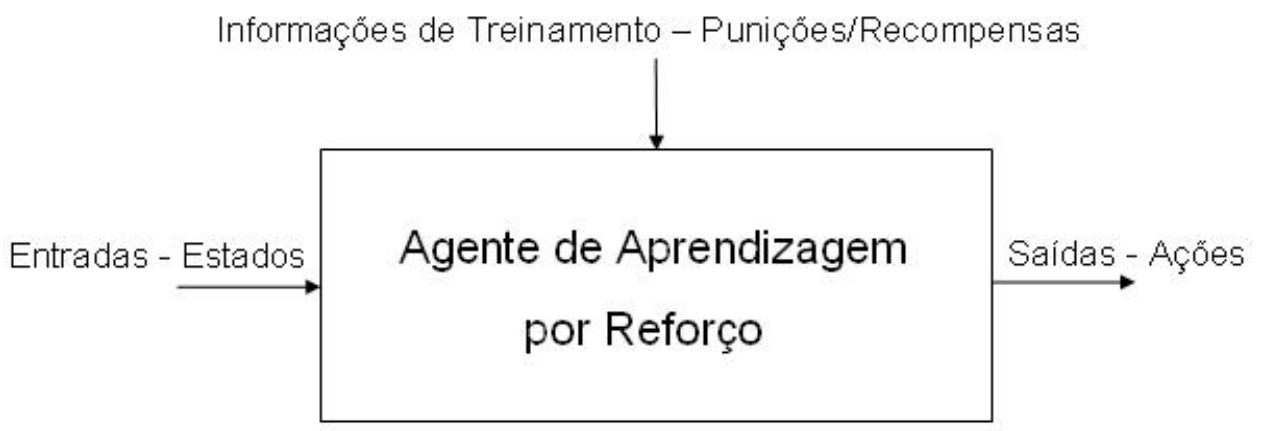

Figura 4.1: Diagrama esquemático de um sistema de aprendizagem por reforço.

O modelo consiste em:

- Um conjunto de estados discretos $S$;

- Um conjunto de ações discretas $A$;

- Informaçoes de treinamento (punições/recompensas).

Neste capítulo, busca-se passar ao leitor informações básicas acerca da aprendizagem por reforço. Inicialmente, na Seção 4.2, apresentam-se algumas características da aprendizagem por reforço. Na Seção 4.3, mostra-se a fundamentação matématica da aprendizagem por reforço, sendo esta baseada na teoria desenvolvida por Markov ([6] e [9]). Em seguida, na Seção 4.4, apresentam-se os elementos que compõem um sistema de aprendizagem por reforço. Finaliza-se o capítulo com a descrição dos principais métodos de aprendizagem por reforço, suas características e diferenças básicas. 


\subsection{Principais Características}

A aprendizagem por reforço apresenta algumas características que lhe são bastante peculiares. Nesta Seção, apresentamos duas características: a maximização do retorno global e o dilema diversificação versus intensificação capazes de distinguir a aprendizagem por reforço de outros métodos de aprendizado não-supervisionado.

\subsubsection{Maximização do Retorno Global}

Uma ação é o produto de uma decisão em um dado estado do ambiente, sendo seu efeito imediato caracterizado por um sinal de reforço. Em sistemas de aprendizagem por reforço busca-se alcançar objetivos globais no ambiente. Denomina-se retorno total, o somatório dos sinais de reforço recebidos pelo agente ao longo de toda interação. Assim, as ações tomadas devem maximi-

zar o retorno total, e não os sinais de reforço recebidos imediatamente após as ações. Deste modo, a qualidade das ações tomadas baseia-se nos valores obtidos no longo prazo. Conseqüentemente, nem sempre a ação que resulta num maior retorno imediato será a melhor ação a ser tomada em um dado estado. Este problema foi tratado por Watkins [10], onde ele conclui que, como a única informação que o agente recebe do ambiente é o reforço imediato, a partir desse valor deve ser gerado algum outro valor que permita obter uma visão da qualidade das ações efetuadas em longo prazo. 


\subsubsection{Diversificação versus Intensificação}

Na aprendizagem por reforço, para cada estado específico, tem-se um conjunto de ações possíveis de serem tomadas. Como será visto mais adiante neste trabalho, associa-se para cada ação possível de ser tomada em um dado estado, um valor, que por sua vez, indica o quão bom é para o agente tomar cada uma dessas ações neste estado, de tal forma que os maiores valores representam as melhores ações (ações gulosas). À medida que o agente visita estados e executa ações, este valor vai sendo atualizado. Sabe-se que o agente deve tomar as ações que maximizem o retorno total. Porém, se o agente sempre toma as melhores ações, a atualização dos valores para as demais ações deste estado não ocorrerá. Em razão disso, várias ações (as denominadas ações não-gulosas) não serão executadas e os valores destas ações não-gulosas nunca serão atualizados. Como conseqüência, o sistema não evoluirá no sentido de alcançar o retorno total máximo. Ele ficará estagnado, tomando sempre as mesmas ações e visitando sempre os mesmos estados. Este problema é conhecido como dilema diversificação versus intensificação, bem conhecido na área de algoritmos genéticos [11].

Para evitar que isso ocorra, algum mecanismo deve ser criado visando permitir o agente visitar todos os estados e tomar todas as ações possíveis. Vários métodos são utilizados na área de aprendizagem por reforço para abordar este dilema, notadamente, a estratégia $\epsilon$-gulosa [6], que será vista mais adiante. Assim, o dilema pode ser resumido como a dificuldade do agente em conciliar a exploração de uma série de possibilidades e, ao mesmo tempo, favorecer progressivamente aquelas que produzem melhores resultados, evitando que ações não sejam visitadas durante o processo de aprendizagem. 


\subsection{Fundamentos matemáticos}

\subsubsection{Propriedade de Markov}

No caso mais geral, a resposta do ambiente em $t+1$ (próximo estado) para uma ação efetuada em $t$ depende de todo o histórico de ações tomadas até o momento atual, ou seja, a dinâmica do ambiente é definida pela especificação completa da distribuição de probabilidades, como mostra a expressão abaixo:

$$
P_{s, s^{\prime}}(a)=\operatorname{Pr}\left\{s_{t+1}=s^{\prime}, r_{t+1}=r \mid s_{t}, a_{t}, r_{t}, s_{t-1}, a_{t-1} \ldots, r_{1}, s_{0}, a_{0}\right\}
$$

onde a probabilidade $(P r)$ do estado $s_{t+1}$ ser o estado $s^{\prime}$ e o reforço $r_{t+1}$ ser igual a $r$, é uma função que depende de todos os estados, ações e reforços passados.

Se a probabilidade de transição de um estado $s$ para um estado $s^{\prime}$ depende apenas do estado $s$ e da ação a adotada em $s$, isso significa que o estado corrente fornece informação suficiente para o sistema de aprendizado decidir qual ação deve ser tomada. Quando o sistema possui essa característica, diz-se que ele satisfaz a propriedade de Markov [9].

Formalmente, a propriedade markoviana é satisfeita se a resposta do ambiente em $t+1$ depende apenas do estado e da ação tomada em $t$. Assim, a probabilidade de transição para o estado $s^{\prime}$ é dada pela expressão:

$$
P_{s, s^{\prime}}(a)=\operatorname{Pr}\left\{s_{t+1}=s^{\prime}, r_{t+1}=r \mid s_{t}=s, a_{t}=a\right\}
$$

A probabilidade de transição do estado $s$ para $s^{\prime}$ satisfaz as condições:

- $P_{s, s^{\prime}}(a) \geq 0$; 


\section{- $\sum_{s^{\prime}} P_{s, s^{\prime}}(a)=1$}

De forma mais simples, o termo $P_{s, s^{\prime}}(a)$ indica a probabilidade de se tomar a ação $a$ no estado $s$ e o próximo estado ser $s^{\prime}$.

A propriedade de Markov, onde as decisões a serem tomadas em um dado instante dependem apenas das informações disponíveis neste instante, permite soluções incrementais, como na programação dinâmica, onde os valores obtidos em um estado $s_{t+1}$ são calculados a partir dos valores obtidos no estado $s_{t}$, de maneira recursiva.

\subsubsection{Processos de Decisão Markovianos - PDM}

Um processo de decisão markoviano ([6] e [9]) é definido como um conjunto de estados $s \in S$ e de ações $a \in A(s)$ e um conjunto de probabilidades $P$ sobre $S$, que representa as probabilidades de transição de estado, de acordo com a Equação 4.2.

De forma análoga, dados o estado atual $s$, uma ação $a$ tomada em $s$ e um estado seguinte $s^{\prime}$, o valor esperado do retorno é:

$$
R_{s, s^{\prime}}^{a}=E\left\{r_{t+1} \mid s_{t}=s, a_{t}=a, s_{t+1}=s^{\prime}\right\}
$$

onde $E$ é o valor esperado do retorno $r_{t+1}$, sempre que o estado $s$, no instante $t$, passe para o estado $s^{\prime}$, no tempo $t+1$, sob a ação $a$.

Os valores de probabilidade $P_{s, s^{\prime}}^{a}$ e retorno esperado $R_{s, s^{\prime}}^{a}$ determinam os aspectos mais importantes da dinâmica de um processo de decisão markoviano finito. Um PDM é dito finito, se o conjunto de estados e ações é finito.

O PDM finito e discreto no tempo pode ser caracterizado da seguinte forma: 
- o ambiente evolui probabilisticamente baseado num conjunto finito e discreto de estados;

- para cada estado do ambiente, existe um conjunto finito de ações possíveis, onde a cada ação é associada uma probabilidade, de tal forma que para as melhores ações são associadas probabilidades maiores de serem escolhidas.

- verifica-se o retorno obtido com cada ação que o sistema de aprendizado executa;

- estados são visitados, ações são executadas e reforços são recebidos.

Finalizando, nem todos os algoritmos da aprendizagem por reforço necessitam de uma modelagem completa do ambiente, ou seja, ter todas as probabilidades de transição $\left(P_{s, s^{\prime}}^{a}\right)$ e valores esperados de retorno $\left(R_{s, s^{\prime}}^{a}\right)$ para todas as transições entre estados possíveis do ambiente. Como exemplo, podemos citar os métodos de aprendizagem por reforço baseados em diferenças temporais [6].

\subsection{Elementos}

Um sistema típico de aprendizagem por reforço constitui-se basicamente em um agente interagindo com seu ambiente (Figura 4.2). O agente percebe situações no ambiente e, baseado nelas, seleciona uma ação para ser tomada. A ação tomada muda de alguma forma o ambiente, e as mudanças são comunicadas ao agente através de um sinal de reforço. Existem quatro partes 
fundamentais na aprendizagem por reforço: a interface agente-ambiente, a política, o reforço e a função de valor.

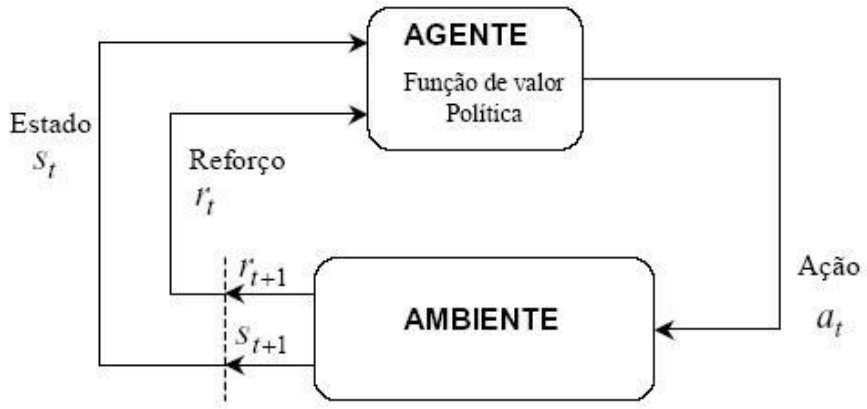

Figura 4.2: Interação entre elementos da aprendizagem por reforço.

No modelo de aprendizagem por reforço utilizado neste trabalho, são apresentadas ao agente percepções de seu ambiente, aos quais aquele responde com ações, sendo estas realizadas sob uma seqüência de instantes de tempo discretos $t_{i}(i=1,2, \cdots, m)$. A cada instante de tempo, o agente observa o estado $s_{t}$ do ambiente e seleciona uma ação $a_{t}$ específica, o que irá provocar uma alteração no estado do ambiente para $s_{t+1}$. Ao realizar a ação $a_{t}$, uma avaliação desta ação, na forma de punição ou recompensa, é apresentada ao agente pelo ambiente. Deste modo, o agente irá interagir com o seu ambiente em busca de otimizar a escolha de suas ações.

O ambiente é representado por um conjunto finito de estados $S$, cujos elementos $s_{t}$ representam os estados tomados no instante de tempo discreto $t_{i}$. Para cada estado, está associado um conjunto $A\left(s_{t}\right)$ finito de ações $a_{t}$. 


\subsubsection{Interface Agente-Ambiente}

Qualquer sistema de aprendizagem por reforço aprende a fazer um mapeamento de situações em ações por meio da interação com um ambiente dinâmico. O ambiente deve ser percebido, ao menos parcialmente, pelo sistema de aprendizado. A informação pode ser do tipo leitura de sensores, descrições simbólicas, entre outras. As ações tomadas podem ser indicações de baixo nível, como valores de tensão, corrente, ou de alto nível, como o movimento de robôs.

\subsubsection{Política}

Uma política, expressa pelo termo $\pi$, representa o comportamento que o sistema da aprendizagem por reforço segue para alcançar o objetivo. Utilizando termos próprios da aprendizagem por reforço, uma política $\pi$ é um mapeamento de estados $s$ em ações $a$, tomadas naquele estado. O valor $\pi(s, a)$ representa a probabilidade de tomar uma dada ação a quando se está no estado $s$.

\subsubsection{Reforço e Retorno}

O reforço é um sinal do tipo escalar representado por $r_{t+1}$ que é devolvido pelo ambiente ao agente, assim que uma ação tenha sido efetuada e uma transição de estado $s_{t} \rightarrow s_{t+1}$ tenha ocorrido. Existem diferentes formas de definir o reforço para cada estado no ambiente, gerando-se funções de reforço que, intrinsecamente, expressam o objetivo que o sistema deve alcançar. $\mathrm{O}$ agente deve maximizar (minimizar) o somatório dos reforços recebidos ao 
longo do tempo, o que nem sempre significa maximizar (minimizar) o reforço imediato a receber, mas o reforço acumulado durante a execução total.

O uso de um sinal de reforço é uma das características que distinguem os métodos de aprendizagem por reforço. Embora o aprendizado baseado em um reforço pareça limitado, foi verificado, na prática, que o uso de reforços é viável, obtendo-se bons resultados [6].

O retorno está relacionado ao valor geral esperado em longo prazo, definido formalmente como um somatório dos reforços obtidos até um tempo t. De modo geral, o sistema busca maximizar (minimizar) o valor esperado de retorno, onde o retorno é definido como uma função da seqüência de valores de reforço até um tempo final $T$. No caso mais simples, as tarefas apresentam um estado terminal e o valor acumulado a longo prazo converge para um valor finito. Estas tarefas são denominadas de horizonte finito (tarefas episódicas) e o retorno é caracterizado de acordo com a seguinte equação:

$$
R_{T}=r_{t+1}+r_{t+2}+r_{t+3}+\ldots+r_{T}
$$

Em muitos casos, a interação entre agente e ambiente não termina naturalmente em um episódio, mas continua sem limite. Por exemplo, em aplicações que exigem um controle contínuo de algum parâmetro, como indústrias de processamento químico. Estas aplicações são chamadas de contínuas, ou tarefas de horizonte infinito. Para elas, a formulação do retorno deve ser alterada pois, caso contrário, seu valor tenderia a infinito (no caso de $r_{t}>0$, $\forall t$ ). Para esta categoria de problemas foi criada a fator de desconto $\gamma$, que torna o cálculo do retorno mais complexo conceitualmente, mas simplifica matematicamente as provas de convergência.

O fator de desconto $\gamma$ determina o grau de influência que os valores futuros 
têm sobre o reforço total, onde $0 \leq \gamma \leq 1$. Os valores de $\gamma$ são determinados de forma empírica, de acordo com cada aplicação [6]. A expressão do retorno para casos de horizonte infinito é:

$$
R_{t}=r_{t+1}+\gamma r_{t+2}+\gamma^{2} r_{t+3}+\ldots=\sum_{k=0}^{\infty} \gamma^{k} r_{t+k+1}
$$

Se $\gamma \rightarrow 0$, o agente tem uma visão míope dos reforços, maximizando (minimizando) apenas os reforços imediatos. Se $\gamma \rightarrow 1$, o agente considera de mesma importância todos os estados futuros.

\subsubsection{Função de Valor}

Uma questão importante na aprendizagem por reforço é saber com certeza se o agente está aprendendo a escolher boas ações, ou seja, ter uma maneira de medir a qualidade das ações que estão sendo tomadas. Função de valor é um número que representa uma estimativa do quão bom é para o agente estar em um dado estado ou tomar uma dada ação em um dado estado seguindo uma política $\pi$ qualquer.

Se uma função de valor considera só o estado $s$, ela é denominada função de valor de estado $V(s)$. Se ela considera o par estado-ação $(s, a)$, então a mesma é denominada função de valor estado-ação e representada por $Q(s, a)$.

Uma vez que os reforços futuros dependem das ações futuras, as funções de valor dependem também da política $\pi$ que o agente segue. Em um processo de decisão markoviano define-se função de valor $V^{\pi}(s)$, dada a política $\pi$, de acordo com a equação abaixo:

$$
V^{\pi}(s)=E_{\pi}\left\{R_{t} \mid s_{t}=s\right\}=E_{\pi}\left\{\sum_{k=0}^{\infty} \gamma^{k} r_{t+k+1} \mid s_{t}=s\right\}
$$


onde a função de valor $V^{\pi}(s)$ é o valor esperado de retorno para o estado $s_{t}=s$ (estado atual), isto é, o somatório dos reforços aplicando-se fator de desconto $\gamma$.

Se considerarmos o par estado-ação, a equação para a função de valor $Q^{\pi}(s, a)$ será a seguinte:

$$
Q^{\pi}(s, a)=E_{\pi}\left\{R_{t} \mid s_{t}=s, a_{t}=a\right\}=E_{\pi}\left\{\sum_{k=0}^{\infty} \gamma^{k} r_{t+k+1} \mid s_{t}=s, a_{t}=a\right\}
$$

Esta equação é semelhante à equação de $V^{\pi}$, sendo, aqui, considerado o valor de retorno esperado para um estado associado a uma ação.

A razão de calcular-se a função de valor para uma dada política $\pi$ é utilizála no auxílio da busca por políticas melhores. Uma política $\pi$ é definida como melhor do que uma política $\pi^{\prime}$ se o valor do seu retorno esperado (Equação 4.3) é maior ou igual ao retorno esperado de $\pi^{\prime}$ para todos os estados $s$. Em outras palavras, $\pi \geq \pi^{\prime}$ se e somente se $V^{\pi}(s) \geq V^{\pi^{\prime}}(s)$ para todo $s \in S$. Existe, no mínimo, uma política, denominada política ótima, que é melhor ou igual a todas as outras políticas. Apesar de poder existir mais de uma política ótima, denotar-se-á esta política por $\pi^{*}$.

Dados uma política e sua função de valor, a troca na política em um estado particular pode ser feita tomando-se a ação que produz o melhor $Q^{\pi}(s, a)$. Este processo permite a origem de políticas glutonas, de modo que a política gerada é tão boa ou melhor que a política original. Esse processo é denominado melhoramento da política, e sempre irá produzir políticas estritamente melhores, salvo se a política $\pi$ já for ótima. Conseqüentemente, em PDM finitos, garante-se a convergência das funções de valor para um valor ótimo, independente da política que está sendo seguida. 
De posse da função de valor estado-ação ótima, $Q^{*}$, é relativamente simples a determinação da política ótima $\pi^{*}$. Para cada estado $s$, existem uma ou mais ações que maximizam (ações gulosas) o valor de $Q$ naquele estado. Deste modo, se $Q^{*}$ é conhecida, as ações que maximizam o valor de $Q$ naquele estado serão as ações ótimas. Uma política ótima irá determinar uma probabilidade não-nula para estas ações gulosas, e uma probabilidade nula para as demais.

\subsection{Métodos}

Para solucionar problemas utilizando-se a abordagem da aprendizagem por reforço, um método de obtenção dos valores de $V^{\pi}$ ou $Q^{\pi}$ deve ser utilizado. Existem três métodos fundamentais de resolução, cada um deles possuindo suas vantagens e desvantagens: programação dinâmica, métodos de Monte Carlo e método de diferenças temporais.

\subsubsection{Programação Dinâmica (Dynamic Programming - DP)}

Programação Dinâmica [9] é uma coleção de algoritmos que pode obter políticas ótimas sempre que existir uma modelagem perfeita do ambiente, ou seja, as probabilidades de transição de estado e os valores esperados dos retornos (ver Equações 4.2 e 4.3). Os algoritmos clássicos de DP são usados de forma limitada pela aprendizagem por reforço, uma vez que a modelagem perfeita do ambiente dificilmente está disponível. Porém, a programação dinâmica oferece um bom fundamento para o conhecimento dos outros méto- 
dos usados na solução de problemas pela abordagem da aprendizagem por reforço.

\subsubsection{Métodos de Monte Carlo (MC Methods)}

Os métodos de Monte Carlo [6] baseiam-se no cálculo da média aritmética de uma amostra de reforços. Para se assegurar que existem valores de retorno bem definidos, métodos MC são utilizados apenas para tarefas episódicas, vale dizer, se assume que a experiência é dividida em episódios que, de algum modo, alcançam o estado final sem depender das ações que foram selecionadas.

O aprendizado é feito a partir de simulações reais com o ambiente, não exigindo, conseqüentemente, o conhecimento a priori das dinâmicas do ambiente, podendo levar, ainda, a um comportamento ótimo. Embora seja necessária uma modelagem, esta requer apenas que algumas transições de estados sejam geradas, sem precisar de todo o conjunto de distribuições de probabilidades para todas as possíveis transições, como é exigido para um modelo que aplica programação dinâmica.

\section{Política $\epsilon$-Gulosa}

Dentro do contexto dos métodos de Monte Carlo e do dilema diversificação versus intensificação, apresenta-se a noção de política $\epsilon$-gulosa [6]. Uma política $\epsilon$-gulosa é uma política que, para cada estado $s \in S$ e ação

$a \in A$, tem-se $\pi(s, a)>0$, significando que a probabilidade de estar num estado $s$ qualquer e tomar uma ação a qualquer é não nula. Mais precisamente, o uso de políticas $\epsilon$-gulosas significa que na maior parte do tempo 
a ação escolhida será aquela que possuir o maior valor de função de valor estado-ação (ação gulosa). Às ações gulosas associa-se uma probabilidade $1-\epsilon+\frac{\epsilon}{|A(s)|}$ de serem escolhidas, onde $|A|$ é o número de ações possíveis de serem realizadas no estado $s$. Para cada uma das demais ações, ditas não

gulosas, associa-se uma probabilidade $\frac{\epsilon}{|A(s)|}$. Em outras palavras, para todas as ações não-gulosas é dado uma probabilidade mínima de ser escolhida. Este processo permite que o algoritmo explore o espaço de estados, e esta é uma das condições necessárias para que se encontre ações ótimas.

\subsubsection{Método de Diferenças Temporais (Temporal Dif- ference Method - TD)}

O método de diferenças temporais resulta da combinação das idéias dos métodos de Monte Carlo com as idéias de programação dinâmica. O aprendizado é feito diretamente a partir da experiência, sem a necessidade de uma modelagem completa do ambiente. Outra característica desse método é a possibilidade de implementação totalmente incremental para aplicações online. Enquanto, nos métodos MC, deve-se aguardar que o ambiente atinja o seu estado final para se obter a atualização das funções de valor, no TD necessita-se aguardar somente até o estado seguinte.

\section{Q-Learning}

Um dos métodos de resolução mais importantes da aprendizagem por reforço é o algoritmo Q-Learning [10]. Tal algoritmo é baseado nos conceitos do método de diferenças temporais e sua convergência para os valores ótimos de $\mathrm{Q}\left(Q^{*}(s, a)\right)$ independe da política que está sendo utilizada. A expressão 
de atualização do valor de $Q$ do algoritmo $Q$-Learning é a seguinte:

$$
Q(s, a) \leftarrow Q(s, a)+\alpha \cdot\left[r_{t+1}+\gamma \max _{a^{\prime}} Q\left(s^{\prime}, a^{\prime}\right)-Q(s, a)\right]
$$

onde $r_{t+1}$ é o retorno associado à transição do estado $s$ para o estado $s^{\prime}, \alpha$ é a taxa de aprendizagem e $\gamma$ é o fator de desconto, com $0 \leq \gamma \leq 1$. A função de valor do estado atual $(Q(s, a))$ é atualizada a partir do seu valor atual, do reforço imediato $\left(r_{t+1}\right)$ e da diferença entre a máxima função de valor no estado seguinte $\left(\max _{a^{\prime}} Q\left(s^{\prime}, a^{\prime}\right)\right)$ e o valor da função de valor do estado atual.

Na solução da equação do Q-Learning, uma questão importante diz respeito ao cálculo do termo $\max _{a^{\prime}} Q\left(s^{\prime}, a^{\prime}\right)$. No caso geral, esse cálculo pode ser visualizado através do diagrama de backup mostrado na Figura 4.3.

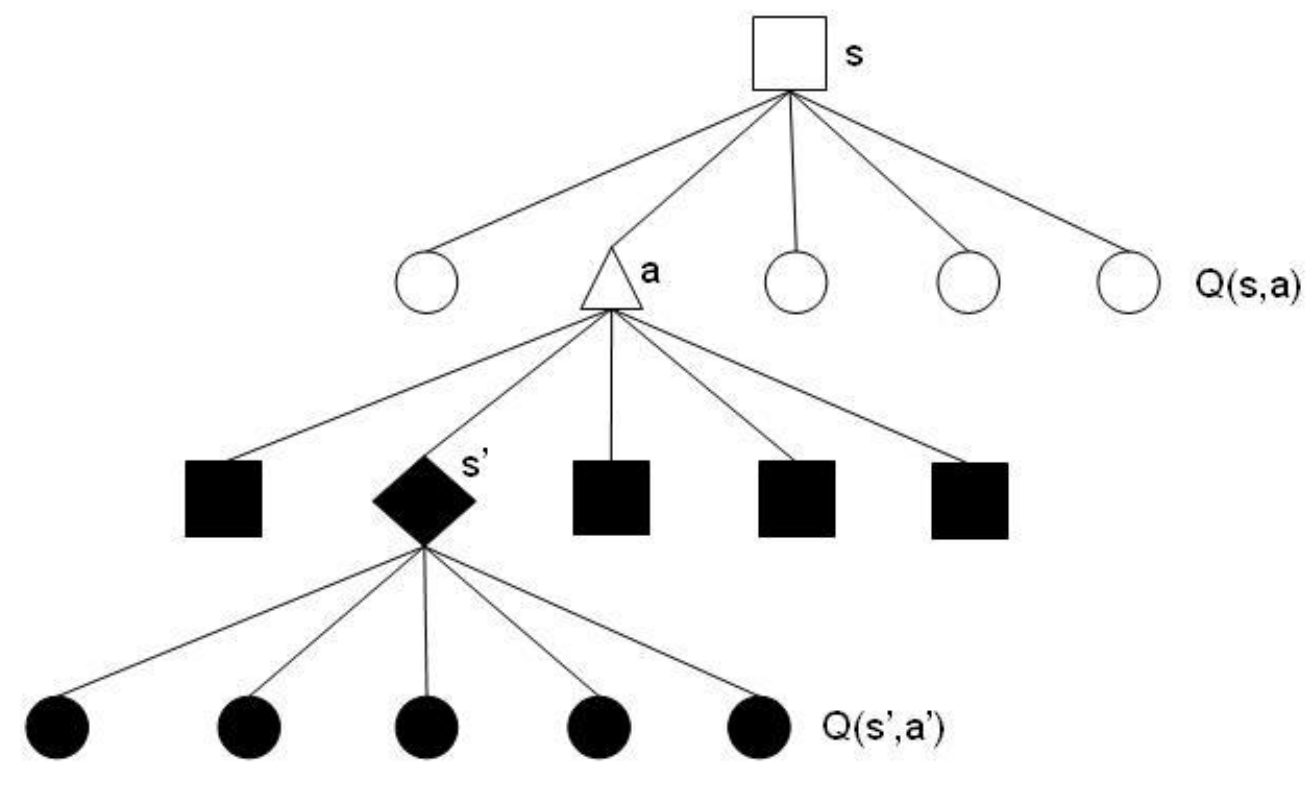

Figura 4.3: Diagrama de backup do algoritmo Q-Learning.

Nessa figura, os estados estão representados por quadriláteros. As ações 
estão representadas por círculos e por um triângulo (ação $a$ ). Uma vez conhecidos $s$ e $a$, tem-se o estado $s^{\prime}$. O valor do termo $\max _{a^{\prime}} Q\left(s^{\prime}, a^{\prime}\right)$ é então tomado entre os valores de $Q\left(s^{\prime}, a^{\prime}\right)$ de todas as ações possíveis de serem tomadas a partir de $s^{\prime}$. Observe-se que não existe a necessidade de se conhecer qual a ação que será tomada em $s^{\prime}$, mas sim quais são todas as ações possíveis de serem tomadas.

Uma característica marcante do Q-Learning é que a função de valor $Q$ aprendida aproxima-se diretamente da função de valor ótimo $Q^{*}$, sem depender da política que está sendo utilizada. Este fato simplifica a análise do algoritmo. A política ainda mantém algum efeito ao determinar qual dos pares estado-ação deve-se visitar e atualizar. A convergência exige que todos os pares estado-ação sejam visitados. Logo, a política $\pi$ a ser utilizada para a determinação do $Q^{*}$ deve ser $\epsilon$-gulosas, pelo menos. O algoritmo $Q$-Learning é apresentado abaixo:

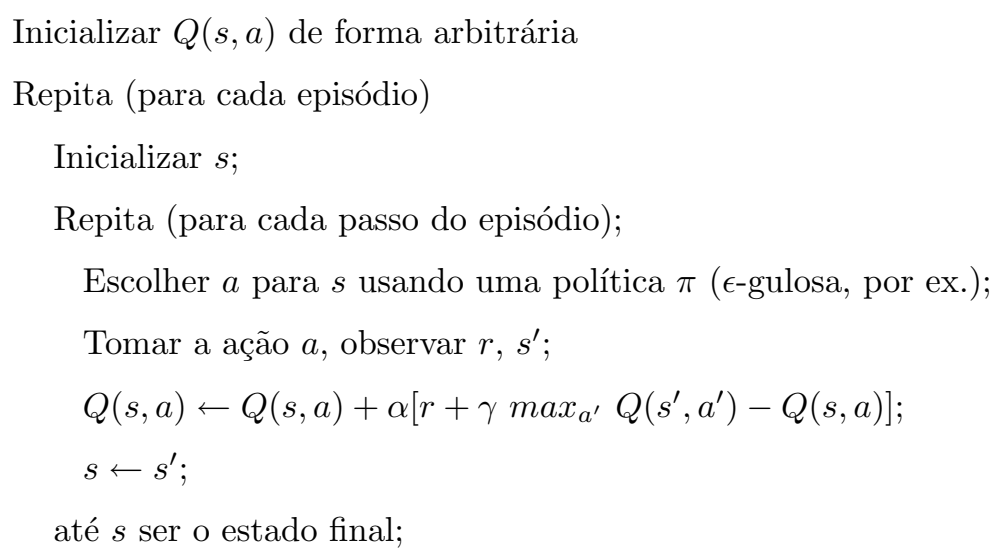

Figura 4.4: Algoritmo Q-Learning. 
O Q-Learning foi o primeiro método de aprendizagem por reforço a possuir fortes provas de convergência [28]. É uma técnica muito simples que calcula diretamente as ações sem avaliações intermediárias e sem uso de modelo. Watkins mostrou que se cada para estado-ação for visitado um número infinito de vezes e com um valor de $\alpha$ adequado, a função de valor $Q$ irá convergir com probabilidade 1 para $Q^{*}$.

A convergência do algoritmo Q-Learning não depende do método de exploração usado. Um agente pode explorar suas ações a qualquer momento. Não existem requisitos para a execução de ações estimadas como as melhores. No entanto, para melhorar o desempenho do sistema é necessário, durante o aprendizado, a busca das ações que maximizem o retorno. 


\section{Capítulo 5}

\section{Aplicação da Aprendizagem por Reforço ao Problema dos k-Servos}

\subsection{Introdução}

A partir de toda a teoria apresentada, o que possibilitou a compreensão do problema abordado e do método de solução a ser usado, foi possível modelar o problema e testar o desempenho da solução proposta. Em termos computacionais, modelar um problema significa implementar a solução visionada de forma algorítmica, utilizando uma linguagem de programação específica. O objetivo de uma modelagem consiste na conversão das informações que foram obtidas na teoria para um formato digital, possiblitando o processamento das informações com o uso de um computador.

Desta maneira e realizando uma modelagem adequada, foi possível a apli- 
cação da aprendizagem por reforço ao problema dos k-servos. O algoritmo Q-Learning foi implementado, utilizando o Matlab 6.0 release $12^{\circledR}$, segundo uma lógica algorítmica, e seu processamento realizado por um computador pessoal.

Neste capítulo, objetiva-se apresentar uma descrição geral sobre a modelagem. Na Seção 5.2, mostra-se a representação de um estado, de acordo com a teoria da aprendizagem por refoço, na implementação. Na Seção 5.3, mostra-se como é feita a atualização dos valores da função de valor Q. Na Seção 5.4, apresenta-se a aplicação do algoritmo Q-Learning. Na Seção 5.5, descreve-se a estrutura de dados utilizada para armazenar os diversos pares estado-ação usados no problema. Finaliza-se o capítulo mostrando os resultados obtidos nos experimentos realizados.

\subsection{Representação dos Estados}

Um estado do ambiente representa uma configuração possível dos servos, ou seja, são $k$-tuplos do tipo $s=\left\{n o_{1}, n o_{2}, \ldots, n o_{k}\right\}$. O número total de estados possíveis é dado pela expressão $\mathcal{C}_{n, k}=\frac{n}{(n-k) !(k) !}$. As ações correspondem aos movimentos permitidos dos servos a partir de um dado estado. Como em um dado estado qualquer um dos servos pode se mover, o número total de ações permitidas por estado é igual a $k$, todas do tipo mover servo localizado no nó $i$ para o nó $j$, de forma a atender a solicitação $\sigma_{i}$. 


\subsection{Q-Learning Aplicado ao Problema dos $k$ - Servos}

Diante do exposto no item precedente, dado um par estado-ação $(s, a)$ e o estado seguinte resultante $s^{\prime}$, deve-se verificar quais são as ações possíveis de serem tomadas a partir de então. Sem perda de generalidade, será considerado que o conjunto de nós é dado por $X=\{1,2, \ldots, n\}$ e que dispõe-se de 3 servos.

Assim sendo, os estados do problema de aprendizagem por reforço são representados por 3-uplos do tipo $s=\left\{n o_{1}, n o_{2}, n o_{3}\right\}$, e dada a solicitação a ser atendida $\sigma_{t}$, o conjunto de ações permitidas em s é $\left\{\right.$ mover_no_1_para_no_ $\sigma_{t}$, mover_no_2_para_no_ $\sigma_{t}$, mover_no_3_para_no_ $\left.\sigma_{t}\right\}$. Se um dos servos encontrase no mesmo local da solicitação $\sigma_{t}$, nenhuma ação é tomada.

Ao se executar a ação de acordo com uma dada política, que pode ser determinística ou $\epsilon$-gulosa, tem-se o estado seguinte $s^{\prime}$. Suponha-se que a ação escolhida foi mover_no_2_para_no_ $\sigma_{t}$. Logo, o estado seguinte será dado por $s^{\prime}=\left\{n o_{-} 1, n o_{-} \sigma_{t}, n o_{-} 3\right\}$. O problema que se coloca é saber quais são todas as ações possíveis de serem tomadas nesse estado. Da mesma forma que antes, elas são \{mover_no_1_para_no_$\sigma_{t+1}$, mover_no_ $\sigma_{t-}$ para $\_$no_$\sigma_{t+1}$, mover_no_3_para_no_ $\left.\sigma_{t+1}\right\}$.

Como não se conhece a priori qual será a solicitação $\sigma_{t+1}$, para se determinar o conjunto de ações permitidas, deve-se considerar todas as possibilidades de movimento dos servos, ou seja, o fato de que $\sigma_{t+1} \in\{1,2, \ldots, n\}$. Um diagrama de backup com todas as ações possíveis em $s^{\prime}$ é mostrado na Figura 5.1. Os conjuntos de ações $a^{\prime}$ estão representados por figuras 
geométricas diferentes, onde cada conjunto representa todas as ações possíveis mover_no_i_para_no_$\sigma_{t+1}$ para um dado $n o_{i}$ e para cada $n o_{-} \sigma_{t+1} \in$ $\{1,2, \ldots, n\}$. Assim, $\max _{a^{\prime}} Q\left(s^{\prime}, a^{\prime}\right)$ deve ser calculado, considerando-se todas essas ações. Formalmente, se $|X|=n$ e existem $k$ servos para atender às solicitações, então existem $k n$ ações possíveis de serem tomadas em $s^{\prime}$, independente de qual seja a solicitação $\sigma_{t+1}$.

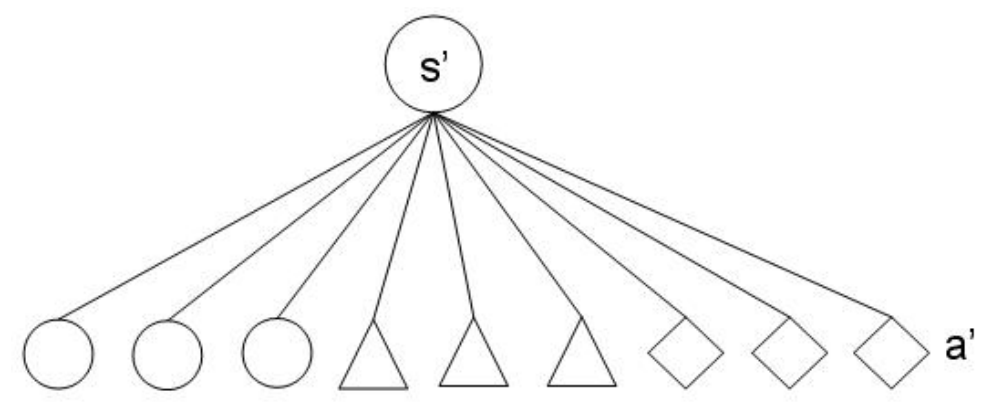

Figura 5.1: Diagrama de backup com todas as ações possíveis em $s^{\prime}$.

Observe-se que, de acordo com o algoritmo do Q-Learning, no instante $t+1$ tem-se $s \rightarrow s^{\prime}$ e, conhecendo-se $\sigma_{t+1}$, as únicas ações $k$ permitidas ${ }^{1}$ são as representadas pelos círculos na Figura 5.1, que correspondem às ações de cada um dos $k$ servos para atender uma requisição no nó $\sigma_{t+1}$.

\subsection{Estrutura de Dados para o Problema}

Como observado na seção anterior, para cada estado do ambiente existem $k n$ ações possíveis. Logo, $Q(s, a)$ pode ser armazenada em uma estrutura de

\footnotetext{
${ }^{1}$ No caso dos problemas de aprendizagem por reforço, em geral, os conjuntos de ações permitidas e ações possiveis são idênticos. No problema dos $k$-servos, esses conjuntos são diferentes, como mostrado na Tabela 5.1.
} 
dimensão $\mathcal{C}_{n, k} \cdot k n$, onde $\mathcal{C}_{n, k}=\frac{n !}{(n-k) ! \cdot k !}$. Esquematicamente, essa estrutura está descrita na Tabela 5.1, considerando-se a existência de $n$ nós e $k$ servos. Dessa forma, os estados têm dimensão $k$ e, conhecendo-se a solicitação $\sigma_{t}$, o número de ações permitidas passa de $k n$ para $k$. Na tabela 5.1, tomou-se por simplicidade um estado $s$ composto de $k$ nós rotulados sucessivamente na forma $s=\{1, \ldots, k\}$. Da mesma forma, a ação denotada por $i \rightarrow 1$ significa, por exemplo, mover o servo do nó $i$ para o nó 1, para atender à solicitação 1 .

Tabela 5.1: Estrutura para o armazenamento dos valores de $Q(s, a)$.

\begin{tabular}{|c|c|c|c|c|c|c|c|}
\hline & \multicolumn{5}{|c|}{ Solicitações } \\
\hline Estado & \multicolumn{3}{|c|}{1} & $\cdots$ & \multicolumn{3}{|c|}{$\mathrm{n}$} \\
\hline$s=\{1, \ldots, k\}$ & $Q(s, 1 \rightarrow 1)$ & & $Q(s, k \rightarrow 1)$ & & $Q(s, 1 \rightarrow n)$ & & $Q(s, k \rightarrow n)$ \\
\hline
\end{tabular}

É fácil verificar que nessa estrutura o cálculo da expressão $\max _{a^{\prime}} Q\left(s^{\prime}, a^{\prime}\right)$ corresponde a encontrar o valor máximo dos elementos da linha associada a um dado $s^{\prime}$, enquanto a escolha da ação associada a uma dada política é obtida a partir do bloco de valores de $Q(s, a)$ indexado por $s$ e $\sigma_{t}$.

\subsection{Resultados}

Para testar o algoritmo desenvolvido neste trabalho, experimentos foram realizados no intuito de observar o desempenho da aprendizagem por reforço em comparação com os algoritmos Harmonic e Work Function, dois dos mais difundidos e estudados métodos de solução do PKS, buscando constatar a eficiência do uso do algoritmo proposto. Foram realizados quatro experimentos, 
cada um deles com espaços métricos de tamanhos distintos e obedecendo, obviamente, a desigualdade triangular. Em cada um destes experimentos, 100 seqüências de requisições por serviço, cada uma delas de tamanho 100, foram geradas. Para cada seqüência de requisições, registrou-se a distância percorrida ao se deslocar os servos, segundo os critérios de seleção estabelecidos em cada algoritmo. Ao final de cada experimento, anotou-se, para cada um dos três algoritmos, a menor e a maior distância percorrida para atender a uma seqüência de pedidos de tamanho 100, o valor médio das distâncias e quantas vezes cada algoritmo foi o melhor em comparação aos demais, vale dizer, quantas vezes conseguiu obter a menor distância percorrida pelos servos para atender a seqüência de 100 requisições. O treinamento do algoritmo da aprendizagem por reforço foi feito a partir de uma seqüência de $3 \cdot 10^{5}$ requisições apresentadas ao mesmo, considerando $\alpha=0.1 \gamma=0.1$ e $\epsilon=0.1$. Os dados registrados nos experimentos são mostrados na Tabela 5.2, para diferentes tamanhos de espaços métricos e quantidade de servos.

Pela análise dos resultados constatou-se que o desempenho do algoritmo proposto neste trabalho obteve, de modo geral, um desempenho mais eficiente nos testes quando comparado aos demais algoritmos. Em seu melhor desempenho, obteve $100 \%$ das vitórias nos testes realizados. Em seu pior desempenho, obteve $79 \%$ das vitórias. Em todos os experimentos, conseguiu obter mais vitórias do que os outros algoritmos. 
Tabela 5.2: Resultados obtidos nos testes.

\begin{tabular}{|c|c|c|c|c|}
\hline \multirow[b]{2}{*}{ Algoritmos } & \multicolumn{4}{|c|}{$n=10 k=2$ iter $=3 e 05$} \\
\hline & Menor & Maior & $\mu$ & Vit. \\
\hline$Q$-Learning & 7454 & 8751 & 8016.30 & 98 \\
\hline Harmonic & 9823 & 11733 & 10806.45 & 0 \\
\hline \multirow[t]{3}{*}{ Work Function } & 7601 & 9200 & 8313.65 & 2 \\
\hline & \multicolumn{4}{|c|}{$n=12 k=2$ iter $=3 e 05$} \\
\hline & Menor & Maior & $\mu$ & Vit. \\
\hline Q-Learning & 7711 & 9342 & 8417.08 & 100 \\
\hline Harmonic & 10191 & 12860 & 11678.44 & 0 \\
\hline \multirow[t]{3}{*}{ Work Function } & 8089 & 9770 & 8818.73 & 0 \\
\hline & \multicolumn{4}{|c|}{$n=15 k=2$ iter $=3 e 05$} \\
\hline & Menor & Maior & $\mu$ & Vit. \\
\hline Q-Learning & 8097 & 9730 & 8779.07 & 79 \\
\hline Harmonic & 8190 & 9698 & 8941.41 & 0 \\
\hline \multirow[t]{3}{*}{ Work Function } & 9951 & 11712 & 10796.38 & 21 \\
\hline & \multicolumn{4}{|c|}{$n=20 k=2$ iter $=3 e 05$} \\
\hline & Menor & Maior & $\mu$ & Vit. \\
\hline Q-Learning & 9090 & 10862 & 9923.28 & 92 \\
\hline Harmonic & 9375 & 11228 & 10164.30 & 1 \\
\hline Work Function & 11212 & 13034 & 12043.72 & 8 \\
\hline Legenda & \multicolumn{4}{|c|}{$\begin{array}{c}\text { Menor - Menor Distância Percorrida } \\
\text { Maior - Maior Distância Percorrida } \\
\text { Vit. - Vitórias Obtidas }\end{array}$} \\
\hline
\end{tabular}




\section{Capítulo 6}

\section{Solução Hierárquica}

\subsection{Introdução}

Como pôde ser visto no capítulo anterior, a estrutura utilizada pela aprendizagem por reforço para armazenar os valores da função de estado-ação $Q$ possui dimensão $\mathcal{C}_{n, k} \cdot k n$, sendo $n$ o número de nós e $k$ o de servos. A dimensão da estrutura se eleva, portanto, em função do número de nós e de servos. Na Figura 6.1, pode-se verificar a dimensão da estrutra para alguns valores de $n$ e $k$.

Ao se analisar o crescimento dos valores da dimensão da estrutura de armazenamento da função $Q$, observa-se que o mesmo ocorre de maneira exponencial à medida que elevamos os valores de $n$. Logo, a complexidade em espaço do algoritmo é $\mathcal{O}\left(k \cdot n^{k+1}\right)$. Isto implica dizer que a solução proposta neste trabalho está limitada pela maldição do dimensionamento (curse of dimensionality ) [9], que significa a impossibilidade de execução de um algoritmo pelo esgotamento de recursos computacionais ou pela inviabilidade 


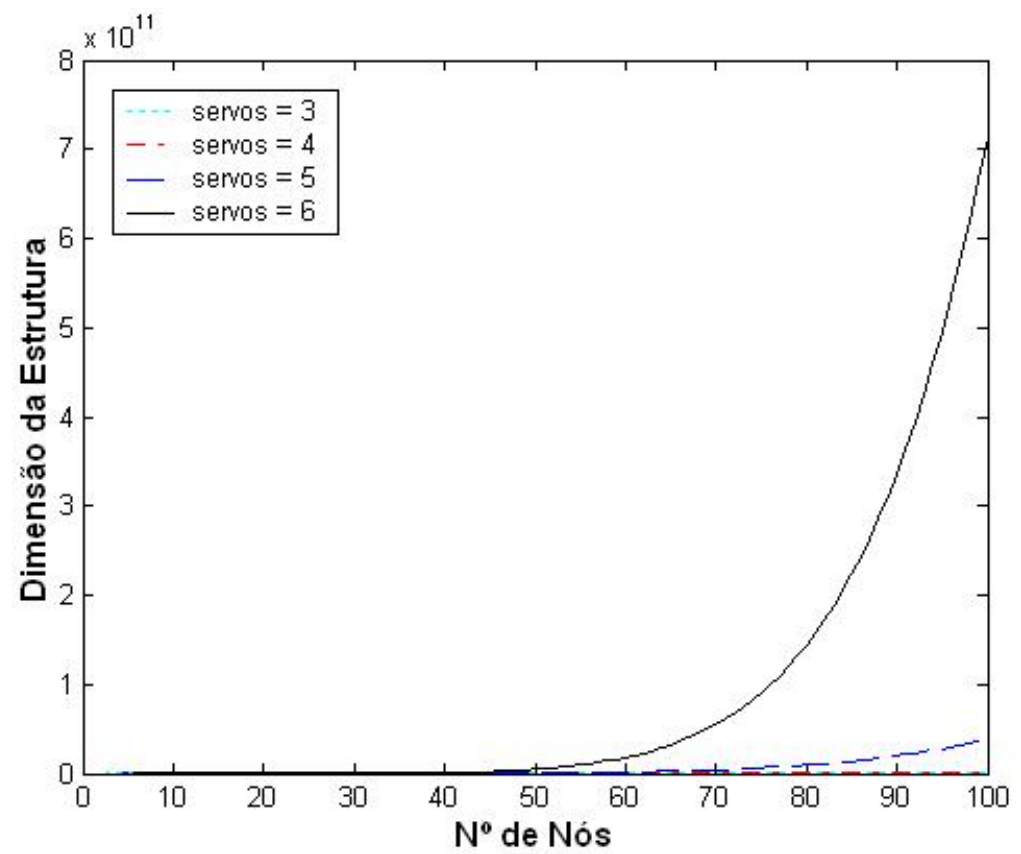

Figura 6.1: Gráfico da dimensão da estrutra de armazenamento da função $Q$ para alguns valores de $n$ e $k$.

do tempo de execução para a obtenção de sua saída. Infere-se, conseqüentemente, que para que se possa aplicar a solução original baseada na aprendizagem por reforço a uma ampla gama de aplicações deve-se criar algum mecanismo que permita a simplificação do problema do curse of dimensionality inerente ao método.

Neste capítulo, a solução para contornar o problema do dimensionamento associado ao Q-Learning será vista. Na Seção 6.2 e em suas subseções será apresentado e explicado em detalhes o algoritmo proposto para solução do problema mencionado. Na Seção 6.3 é apresentado a análise formal da complexidade do algoritmo. Finaliza-se o capítulo na Seção 6.4, onde são mostra- 
dos os resultados obtidos nos testes realizados para comprovar a eficiência da solução.

\subsection{Apresentação da Solução Hierárquica}

Para contornar o problema do dimensionamento, uma solução hierarquizada baseada na aprendizagem por reforço e no método guloso foi proposta. A idéia geral é aplicar a aprendizagem por reforço a um número reduzido de nós, selecionados seguindo um critério específico, no espaço métrico do problema e tentar generalizar o aprendizado obtido neste treinamento para outros pares estado-ação não visitados. Quando a generalização não for possível, utiliza-se o critério guloso para selecionar o servo a ser deslocado. Sucintamente, os passos de execução do algoritmo hierárquico são os seguintes:

1. Divide-se o conjunto de nós em grupamentos de proximidade.

2. Para cada grupamento formado, escolhe-se um nó para representar o grupo.

3. Executa-se o algoritmo da aprendizagem por reforço no conjunto de nós-centro escolhidos.

4. Para cada grupamento formado, executa-se o algoritmo da aprendizagem por reforço no conjunto de nós que compõem o grupo.

5. Para cada solicitação, se o par estado-ação não foi visitado nos passos anteriores, utiliza-se o critério guloso para escolher o servo a ser deslocado. 
Cada um dos passos listados acima e a apresentação do algoritmo em português estruturado será explicado minuciosamente nas subseções a seguir.

\subsubsection{Divisão em Grupamentos de Proximidade}

A divisão do espaço métrico em grupamentos foi feita utilizando-se o algoritmo de Boruvka. Neste algoritmo, a divisão do grafo em grupos é feita a partir de sua Árvore Geradora Mínima (AGM). Uma árvore geradora mínima de um grafo $G=(N, A)$ é um subgrafo ${ }^{1}$ conexo $^{2}$ e acíclico ${ }^{3}$ de $\mathrm{G}$ que contém todos os nós do grafo e cuja soma do comprimento de suas arestas é mínimo em G.

O funcionamento do algoritmo de Boruvka é bastante simples. Inicialmente, o número de grupos é igual ao de nós que compõem o grafo a ser dividido, ou seja, cada nó irá representar um grupo. O algoritmo irá iteragir realizando a fusão entre grupos até que a quantidade obtida de grupos seja igual à desejada pelo usuário, o qual será armazenada na variável ENT e será fornecida como um parâmetro de entrada pelo usuário. Esta fusão é feita levando-se em consideração a distância entre dois grupos. Para cada grupo $i$, observa-se o conjunto de arestas que ligam $i$ aos seus vizinhos (diz-se que dois grupos são vizinhos ou adjacentes se existe uma aresta ligando-os diretamente). Em seguida, verifica-se qual aresta possui a menor distância a um grupo adjacente a $i$. Finalmente, o grupo $i$ e o adjacente mais próximo,

\footnotetext{
${ }^{1}$ Um subgrafo $G_{1}=\left(N_{1}, A_{1}\right)$ de $G$ é um grafo tal que $N_{1} \subset N$ e $A_{1} \subset A$.

${ }^{2} \mathrm{Um}$ grafo $G=(N, A)$ é dito conexo se existe um caminho entre cada par de vértices de $G$.

${ }^{3} \mathrm{Um}$ grafo $G=(N, A)$ é acíclico se não não existe nenhum caminho fechado, que começa e termina no mesmo vértice, ligando seus vértices.
} 
o da aresta com menor distância, se fundem e dão origem a um novo grupo.

A apresentação do algoritmo em português estruturado pode ser visualizada na Figura 6.2.

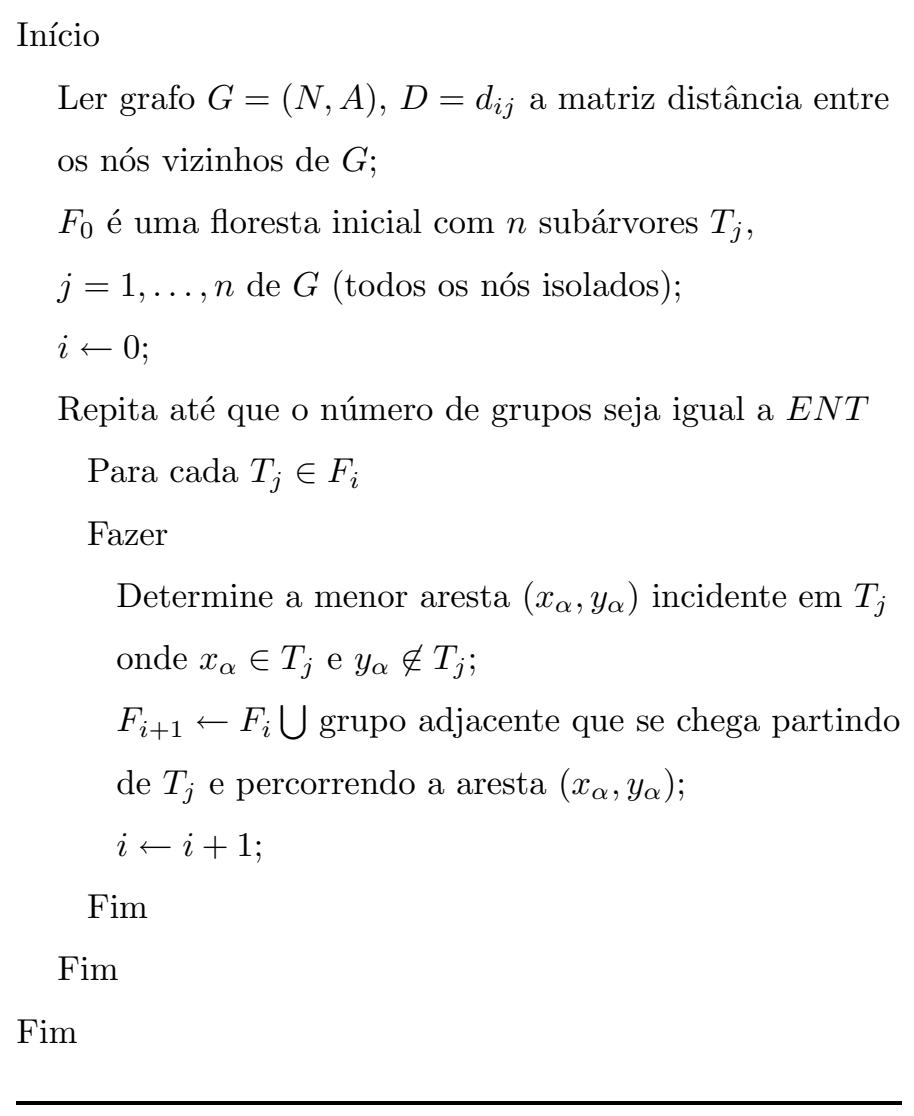

Figura 6.2: Algoritmo de Boruvka. 


\subsubsection{Seleção dos Nós - Escolha dos Centros dos Gru- pamentos}

De posse do conjunto de grupamentos formados, o próximo passo é selecionar os nós que irão representar cada um destes grupos. Denominou-se os nós selecionados de nós-centro. O critério de escolha destes nós é a média da sua distância em relação aos demais nós que compõem seu grupo. Em outras palavras, o nó selecionado será aquele que possuir, em média, a menor distância em relação a todos os outros nós que compõem seu grupo. O algoritmo em português estruturado que implementa a escolha dos nós-centro pode ser visto na Figura 6.3.

Na Figura 6.3, menor_media armazena o valor do nó que obteve a melhor média em seu grupo, sendo, portanto o nó selecionado. A variável acum armazena o valor da média para cada nó do conjunto de nós.

\subsubsection{Execução da Aprendizagem por Reforço no Con- junto Reduzido de Nós}

Devido ao problema do curse of dimensionality, a aprendizagem por reforço não pode ser aplicada a problemas de maior porte devido a inviabilidade do tempo computacional para a execução do algoritmo. Entretanto, para problemas de menor porte, verificou-se, em capítulos anteriores deste trabalho, que a técnica do aprendizado por reforço se comporta de maneira mais eficiente em relação a outros algoritmos. A idéia por trás da criação deste algoritmo hierárquico é a aplicação da aprendizagem por reforço a um número reduzido de nós em relação ao conjunto de nós do problema. Em seguida, 


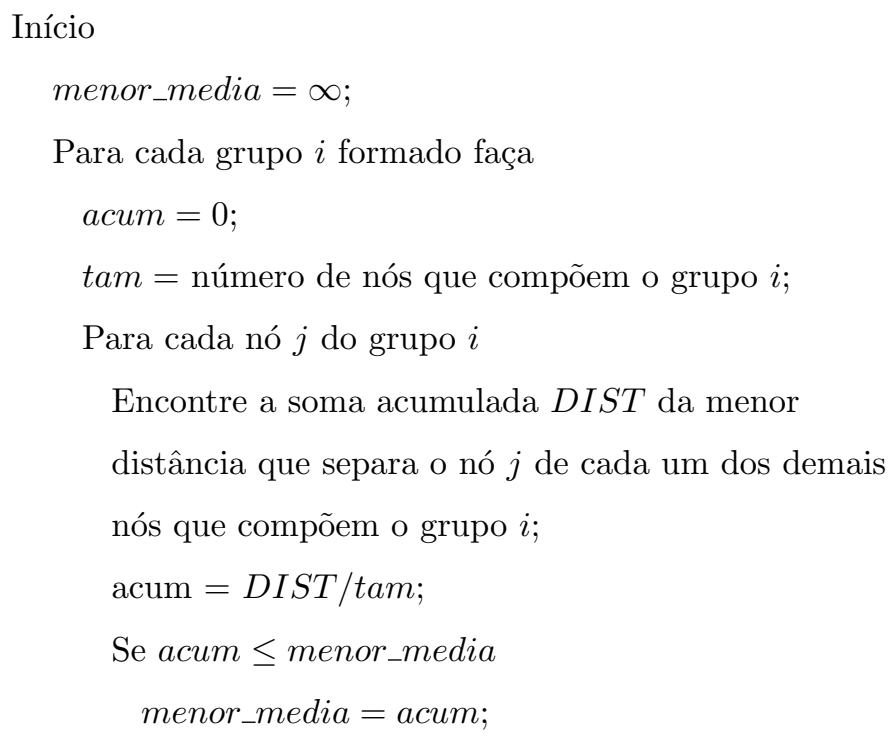

Figura 6.3: Algoritmo de seleção dos nós-centro.

busca-se a generalização do aprendizado obtido a outros pares estado-ação não visitados. Quando não puder ser utilizado nem o aprendizado nem a generalização, o critério guloso será utilizado para escolher qual servo será deslocado.

Nos primeiros passos do algoritmo hierárquico, o conjunto de $n$ nós do problema foi dividido em $x$ grupamentos de proximidade (onde, $x \leq n$ ). Para cada grupamento um nó-centro foi selecionado, totalizando $x$ nós-centro. Cada um destes $x$ grupamentos contém um determinado número de nós ${ }^{4}$, de

\footnotetext{
${ }^{4}$ Cada grupo deve conter pelo menos 1 nó. Desta forma, o número de nós por grupo
} 
tal forma que o somatório dos nós que compõem os $x$ grupos é igual a $n$.

A aprendizagem por reforço será aplicada no conjunto de $x$ nós-centro e em cada conjunto de nós que compõem os $x$ grupamentos, mantendo-se constante o total de $k$ servos. A execução da aprendizagem por reforço, neste conjunto reduzido de nós, mantém as mesmas características da execução no conjunto de $n$ nós do problema. Porém, deve-se observar que os servos e os possíveis locais de demanda estarão localizados somente nos nós selecionados para cada execução.

Assim, durante a execução da aprendizagem por reforço no conjunto de $x$ nós-centro, os servos só poderão se deslocar e as demandas só poderão surgir nestes nós. Do mesmo modo, durante a execução em um dos $x$ grupamentos de proximidade, os servos e as demandas só estarão localizados nos nós que compõem o grupo.

\subsubsection{Implementação do Algoritmo Hierárquico}

A implementação do algoritmo hierárquico em português estruturado pode ser visualizada na Figura 6.4.

Os passos do algoritmo poderão ser melhor visualisados com a compreensão do seguinte exemplo. Considere um problema, o qual denominaremos de original, com 50 nós e 2 servos. A dimensão da estrutura de armazenamentos da função $Q$ do problema é dado pela expressão $\mathcal{C}_{50,2} \cdot 50 \cdot 2=$ $1225 \cdot 20 \cdot 2=122500$. Primeiramente, o conjunto de nós é dividido em 10 grupos, e em cada grupo um nó-centro é selecionado, totalizando 10 nóscentro. Agora, a dimensão do problema com um número inferior de nós é pode variar entre 1 e $n-x+1$. 
Encontre $x$ grupamentos utilizando o Algoritmo de Boruvka;

Para cada grupamento $x$ formado

Execute o Q-Learning no conjunto de nós que compõem o grupo;

Selecione o nó que será o centro do grupamento em questão;

Fim

Execute o $Q$-Learning nos $x$ nós-centro selecionados e com $k$ servos e encontre a política $\pi$;

Para cada requisição $\sigma_{i}$

Se o par estado-ação foi visitado pelo Q-Learning

O servo a ser deslocado será determinado pela política $\pi$;

Senão

Se o grupamento da demanda for o mesmo que o de um ou mais servos

Selecione os servos que pertencem ao mesmo grupo da demanda;

Dentre os servos selecionados, desloque o que estiver mais próximo à demanda;

Senão

Se o grupamento da demanda for diferente do grupamento dos $k$ servos

Se os $k$ servos pertencem ao mesmo grupamento

Desloque o servo que estiver mais próximo à demanda;

Senão

Se os k servos pertencem a grupamentos distintos

Considere que cada servo e a demanda estão localizados nos centros dos seus

grupamentos;

O servo a ser deslocado será determinado pela política $\pi$;

Senão

O servo a ser deslocado será o que estiver mais próximo à demanda;

Fim_do_algoritmo

Figura 6.4: Algoritmo Hierárquico. 
igual a $\mathcal{C}_{10,2} \cdot 10 \cdot 2=45 \cdot 10 \cdot 2=900$. A aprendizagem por reforço será executada:

- No conjunto dos 10 nós-centro selecionados;

- Em cada um dos $x$ conjuntos de nós que compõem os grupamentos formados.

Em ambos os casos, mantêm-se constante o número de servos (número de servos igual a 2). A política $\pi$ correspondente aos pares estado-ação visitados será obtida. Os deslocamentos dos servos e os locais de demanda só ocorrerão:

- Quando a aprendizagem por reforço for executada nos 10 nós-centro selecionados, os deslocamentos e o surgimento de demandas só ocorrerão em pontos situados nestes nós-centro;

- Quando a aprendizagem por reforço for executada dentro de cada um dos 10 grupamentos formados, os deslocamentos e o surgimento de demandas só ocorrerão em pontos situados nos nós que compõem cada grupamento.

Considere cinco exemplos de possíveis localizações de servos e surgimento de requisições por serviço, considerando o problema original, e a correspondente solução ao se seguir os passos do algoritmo hierárquico:

1. Os 2 servos estão localizados em nós-centro distintos e surge uma demanda em um outro nó-centro: como o par estado-ação já foi apresentado durante a fase de execução da aprendizagem por reforço, o servo deslocado será aquele que possuir o maior valor da política $\pi$ para o respectivo par estado-ação. 
2. Os 2 servos estão localizados em nós-centro distintos e surge uma demanda em um local, que não é nó-centro, num grupo distinto ao dos servos: o algoritmo considerará que a demanda está inserida no nócentral de seu grupo. Com isso, este par estado-ação com a demanda transposta ao nó-centro já foi visitado durante o aprendizado, implicando a seleção do servo que apresentar o maior valor para a política $\pi$ correspondente ao estado-ação. Obviamente, no cômputo da distância percorrida pelo servo será considerado o deslocamento do mesmo até o local original da demanda e não o do nó-centro considerado.

3. Os 2 servos estão localizados em grupos distintos e surge uma demanda num nó que pertence ao grupo de um deles: o servo a ser deslocado será aquele que pertencer ao grupo da demanda. Se por acaso fosse 3 servos, e 2 deles pertencessem ao grupo da demanda e 1 não, o deslocado seria aquele que pertencesse ao grupo da demanda e que estivesse mais próximo a mesma (critério guloso).

4. Os 2 servos estão localizados em um mesmo grupo: independente de onde estiverem localizados os servos e a requisição por serviço, o servo a ser deslocado será escolhido pelo método guloso. Vale dizer, o servo mais próximo à demanda atenderá o pedido.

5. Os 2 servos estão em grupos distintos, um deles está num nó-centro e o outro não, e surge uma demanda em um nó que não é centro e que pertence a um grupo que é distinto ao dos 2 servos: tanto o servo quanto a demanda serão consideradas como se estivessem localizados no nó-central do seu grupo. Este estado transposto já foi visitado durante 
o aprendizado e o servo escolhido será o que apresentar o maior valor para a política $\pi$ correspondente. Mais uma vez, os deslocamentos serão calculados segundo as posições originais dos servos e da demanda.

Deve-se observar que o conjunto de todos os pares estado-ação que compõem o problema original não serão visitados quando utilizado o algoritmo hierárquico. Entretanto, esta restrição pode ser contornada através do uso de uma estratégia denominada generalização (o qual será explicado mais adiante).

Do exposto, infere-se que quando os servos e as demandas pertencerem a grupos distintos, todos eles, e os mesmos não estiverem nos nós-centrais dos seu respctivos grupos, serão considerados como se estivessem. A partir desta suposição, utiliza-se o conhecimento obtido durante a execução da aprendizagem por reforço e se escolhe o servo a ser deslocado. Esta transposição da posição dos servos ou da requisição faz com que o aprendizado feito na execução da aprendizagem por reforço possa ser utilizado em pares estado-ação que não foram visitados, generalizando para outros estados o conhecimento obtido durante o aprendizado.

\subsection{Complexidade do Algoritmo Hierárquico}

A redução da complexidade do algoritmo hierárquico em relação a abordagem da aprendizagem por reforço que considera o conjunto completo de nós ocorre em função de um parâmetro $\delta$, denominado fator de redução. $\mathrm{O}$ tamanho da estrutura de armazenamento necessária para armazenar os valores da função $Q$ também irá ocorrer em função de $\delta$, sendo este número 
igual a $\mathcal{C}_{\frac{n}{\delta}, k} \cdot k \cdot \frac{n}{\delta}$, onde $\mathcal{C}_{\frac{n}{\delta}, k}=\frac{\left(\frac{n}{\delta} !\right)}{\left(\frac{n}{\delta}-k\right) ! \cdot k !}$. Através deste número e realizando as manipulações necessárias, obter-se-á $\mathcal{O}=\frac{1}{(\delta)^{k}} \cdot n^{k}$ como complexidade. Portanto, a redução da complexidade em relação ao problema original é da ordem de $\frac{1}{(\delta)^{k}}$.

\subsection{Resultados}

Para testar o algoritmo hierárquico desenvolvido neste trabalho, duas comparações foram realizados no intuito de observar o desempenho do mesmo. A primeira foi feita com o algoritmo Q-Learning, considerando espaços métricos cujos tamanhos pudessem ser processados sem maiores problemas pela aprendizagem por reforço. Claramente, o desempenho alcançado pelo algoritmo hierárquico não seria superior, nem mesmo equivalente, ao Q-Learning, por utilizar o critério guloso que, comprovadamente, não garante soluções ótimas. Contudo, um desempenho inferior em uma porcentagem que não fosse expressiva poderia mostrar que a solução hierárquica poderia ser satisfatória se comparada com os algoritmos, cujo desempenho é inferior ao Q-Learning, que são utilizados para solucionar problemas de maior porte. Para os testes, 100 seqüências de requisições por serviço, cada uma delas de tamanho $500^{5}$, foram geradas. Em cada experimento, registrou-se a distância percorrida ao se deslocar os servos, segundo os critérios de seleção estabelecidos em cada algoritmo. No término dos experimentos, anotou-se, para cada algoritmo, a

\footnotetext{
${ }^{5}$ Cada seqüência de requisições por serviço de tamanho 500 foi denominada de experimento. Pode-se dizer, portanto, que 100 experimentos foram realizados para cada tamanho de espaço métrico.
} 
menor e a maior distância percorrida para atender à seqüência de pedidos de tamanho 500, o valor médio das distâncias e o valor médio, em porcentagem, do quão inferior foi o algoritmo hierárquico em relação ao Q-Learning, melhor dizendo, somou-se a distância total percorrida nos 100 experimentos pelos dois algoritmos e verificou-se a diferença percentual entre estes valores. Este percentual está indicado na última coluna da Tabela 6.1. O treinamento do algoritmo da aprendizagem por reforço foi feito a partir de uma seqüência de $3 \cdot 10^{5}$ requisições apresentadas ao mesmo, considerando $\alpha=0.1 \gamma=0.9$ e $\epsilon=0.1$. Os dados registrados nos experimentos são mostrados na Tabela 6.1. 
Tabela 6.1: Resultados experimentais da comparação entre os algoritmos Hierárquico e Q-Learning.

\begin{tabular}{|c|c|c|c|c|c|}
\hline \multirow[b]{2}{*}{ Algoritmos } & \multicolumn{5}{|c|}{$n=18 k=2$ iter $=3 e 05$} \\
\hline & Min & $\operatorname{Max}$ & Média & SD & $\%$ \\
\hline Q-Learning & 12529 & 14327 & 13345.47 & 384.40 & - \\
\hline Hierárquico & 14596 & 16833 & 15659.78 & 442.76 & 17.34 \\
\hline \multirow[b]{2}{*}{ Algoritmos } & \multicolumn{5}{|c|}{$n=20 k=2$ iter $=3 e 05$} \\
\hline & Min & $\operatorname{Max}$ & Média & SD & $\%$ \\
\hline Q-Learning & 10977 & 13189 & 12022.92 & 417.59 & - \\
\hline Hierárquico & 11800 & 13694 & 12787.57 & 429.84 & 6.36 \\
\hline \multirow[b]{2}{*}{ Algoritmos } & \multicolumn{5}{|c|}{$n=24 k=2$ iter $=3 e 05$} \\
\hline & Min & $\operatorname{Max}$ & Média & SD & $\%$ \\
\hline Q-Learning & 13239 & 15282 & 14158.92 & 414.34 & - \\
\hline Hierárquico & 14006 & 15906 & 15017.80 & 439.50 & 6.70 \\
\hline Legenda & \multicolumn{5}{|c|}{$\begin{array}{l}\text { Min - Menor distância percorrida no teste } \\
\text { Max - Maior distância percorrida no teste } \\
\% \text { - Inferioridade percentual do hierárquico }\end{array}$} \\
\hline
\end{tabular}

Pela análise dos resultados da Tabela 6.1, verificou-se que o desempenho do algoritmo hierárquico não foi expressivamente inferior ao do Q-Learning nos experimentos realizados.

No segundo teste, buscou-se constatar a aptidão do algoritmo hierárquico 
em solucionar o PKS para problemas de maior porte de maneira eficiente. O objetivo era verificar se o mesmo seria capaz de solucionar problemas mais robustos consumindo um tempo computacional viável e a sua eficiência em comparação ao algoritmo Harmonic, já que o mesmo não é afetado pelo problema da explosão computacional. Em função disso, resolveu-se um problema com um conjunto de nós de tamanho 100 e com 3 servos. Variou-se a quantidade de grupamentos formados para verificar se existia alguma relação com o desempenho obtido. Para os testes, 100 seqüências de requisições por serviço, cada uma delas de tamanho 500, foram geradas. Para cada seqüência de requisições, registrou-se a distância percorrida ao se deslocar os servos, segundo os critérios de seleção estabelecidos em cada algoritmo. Ao final de cada experimento, anotou-se, para cada algoritmo, a menor e a maior distância percorrida para atender a uma seqüência de pedidos de tamanho 500, o valor médio das distâncias e quantas vezes cada algoritmo foi o melhor em comparação ao outro, vale dizer, quantas vezes conseguiu obter a menor distância percorrida pelos servos para atender a seqüência de 500 requisições. O treinamento do algoritmo da aprendizagem por reforço nos nós-centro foi feito a partir de uma seqüência de $3 \cdot 10^{5}$ requisições apresentadas ao mesmo, considerando $\alpha=0.1 \gamma=0.9$ e $\epsilon=0.1$. Os dados registrados nos experimentos são mostrados nas Tabela 6.2.

Pela análise dos resultados acima, verificou-se que os resultados obtidos pelo algoritmo hierárquico foram melhores que os obtidos pelo Harmonic. Não foi observada nenhuma relação entre o desempenho alcançado pelo algoritmo hierárquico e o número de grupamentos selecionados pelo mesmo. 
Tabela 6.2: Resultados experimentais da comparação entre os algoritmos Hierárquico e Harmonic.

\begin{tabular}{|c|c|c|c|c|c|}
\hline \multirow{2}{*}{ Algoritmos } & \multicolumn{5}{|c|}{$n=100 k=3$ grupos $=18$ iter $=3 e 05$} \\
\cline { 2 - 6 } & Min & Max & Média & SD & Vitórias \\
\hline Hierárquico & 6010 & 6794 & 6421.53 & 156.98 & 65 \\
\hline Harmonic & 6045 & 6879 & 6465.77 & 151.81 & 35 \\
\hline \multirow{3}{*}{ Algoritmos } & $n=100 k=3$ grupos $=20$ iter $=3 e 05$ \\
\cline { 2 - 6 } & Min & Max & Média & SD & Vitórias \\
\hline Hierárquico & 6096 & 6867 & 6403.64 & 145.56 & 80 \\
\hline Harmonic & 6176 & 6884 & 6498.21 & 136.54 & 20 \\
\hline \multirow{3}{*}{ Algoritmos } & $n=100 k=3$ grupos $=24$ iter $=3 e 05$ \\
\cline { 2 - 6 } & Min & Max & Média & SD & Vitórias \\
\hline Hierárquico & 2055 & 3104 & 2517.07 & 216.13 & 75 \\
\hline Harmonic & 2370 & 3699 & 2980.03 & 241.95 & 25 \\
\hline Legenda & \multicolumn{5}{c}{ Média - média dos custos } \\
& \multicolumn{6}{|c|}{ SD - Standard Deviation } \\
& Min - Menor distância percorrida no teste \\
& Max - Maior distância percorrida no teste \\
\hline
\end{tabular}




\section{Capítulo 7}

\section{Considerações Finais}

\subsection{Conclusão}

O objetivo do presente trabalho consiste em verificar a adequabilidade da aprendizagem por reforço na solução do problema dos k-servos. Para tanto, modelou-se o problema como um processo de decisão markoviano e aplicou-se o algoritmo Q-Learning. Para testar o algoritmo desenvolvido neste trabalho, experimentos foram realizados no intuito de observar o desempenho da aprendizagem por reforço. Através da comparação entre os resultados obtidos nos experimentos pela aprendizagem por reforço e os algoritmos Harmonic e Work Function, dois dos mais difundidos e estudados métodos de solução do PKS, constatou-se, empiricamente, a eficiência do uso do algoritmo proposto. A partir de espaços métricos de tamanhos variados, e de seqüências de requisições de tamanhos pré-fixados em 100, ambos gerados aleatoriamente e devendo o espaço métrico obedecer a desigualdade triangular, anotou-se, para cada um dos três algoritmos, a menor e a maior 
distância percorrida para atender a uma seqüência de pedidos de tamanho 100, o valor médio das distâncias e quantas vezes cada algoritmo foi o melhor em comparação aos demais, vale dizer, quantas vezes conseguiu obter a menor distância percorrida pelos servos para atender a seqüência de 100 requisições. Implementou-se, também, um problema de paginação para comprovar a eficiência da aprendizagem por reforço em aplicações práticas do PKS. Porém, ainda se faz necessário a aplicação do algoritmo desenvolvido a instâncias de problemas reais, para que se comprove, a aplicabilidade desta solução em casos concretos.

A abordagem da aprendizagem por reforço pode ser facilmente extendida a problemas correlatos ao k-servos, já que, como foi visto, a mesma apresentou desempenho satisfatório em relação aos algoritmos Harmonic e Work Function. Esta abordagem pode ser aplicada a qualquer espaço métrico, eliminando a restrição de se usar somente espaços métricos que obedeçam a desigualdade triangular (esta restrição é imposta pelo algoritmo Work Function). Pode-se verificar, ainda, a modelagem e o desempenho do mesmo em relação às mais diversas aplicações do PKS.

Entretanto, o problema do curse of dimensionality inerente ao método, impossibilita a aplicação da solução original baseada na aprendizagem por reforço a aplicações onde o número de nós seja elevado devido a inviabilidade do tempo de processamento da solução, limitando sua aplicação a problemas de menor porte, onde o número de possíveis locais de demanda e de servos é reduzido.

Para evitar esta restrição, uma nova solução, denominada hierárquica, foi proposta. Esta solução utiliza dois métodos de solução do PKS, a apren- 
dizagem por reforço, aplicada a um número reduzido de locais de demanda, e o método guloso, onde o critério de escolha do agendamento dos servos é baseado na menor distância ao local de demanda.

Para verificar o desempenho da solução hierárquica, dois novos testes foram realizados. No primeiro, verificou-se, em um problema que pudesse ser processado viavelmente pela solução baseada exclusivamente na aprendizagem por reforço, o quão inferior é o desempenho da solução hierárquica em relação ao Q-Learning. A razão óbvia da inferioridade da solução hierárquica em detrimento ao Q-Learning se deve ao fato daquele utilizar o método guloso na escolha dos deslocamentos dos servos em alguns casos, sendo o método guloso, comprovadamente, não garantidor da solução ótima. No segundo, aplicado a um problema de maior porte, analisou-se o seu desempenho em comparação com o algoritmo Harmonic, método de solução do PKS não restrito ao problema do dimensionamento, amplamente difundido e cuja complexidade, comprovada formalmente [22], é superior a outros métodos de soluções para problemas de maior porte.

Em ambos os testes, verificou-se que o desempenho da solução hierárquica é satisfatório, superando, inclusive, o desempenho do algoritmo Harmonic no problema de maior porte apresentado.

\subsection{Perspectivas de Trabalhos Futuros}

A adequação da solução hierárquica a problemas reais ainda precisa ser verificada. Um bom parâmetro de comparação do desempenho do algoritmo proposto para um problema de otimização de sondas de produção terrestre 
pode ser visto em [32].

O uso de redes neurais para a interpolação dos valores da função $Q$ também precisa ser verificada [33]. As implicações do uso desta estratégia, caso a mesma seja viável, permitiria a interpolação dos valores de $Q$ para pares estado-ação não visitados durante a execução da aprendizagem por reforço.

Estudos relacionados à verificação formal dos resultados precisam ser feitos, no intuito de se demonstrar, matematicamente, a competitividade da solução apresentada. 


\section{Referências Bibliográficas}

[1] Manasse, M. S., McGeoch, L. A. e Sleator, D. D., Competitive Algorithms for On-Line Problems, In: Proc. 20th Annual ACM Symposium on Theory of Computing, pags. 322-33, 1988.

[2] Sleator, D. e Tarjan, R., Armotized Efficiency of List Update and Paging Rules. Communications of the ACM, 28(2):202-208, 1985.

[3] A., Floratos e R., Boppana, The On-line K-server Problem, Courant Institute of Mathematical Sciences, NYU.

[4] P., Raghavan e M., Snir, Memory Versus Randomization in On-Line Algorithms, Proc. 16th International Colloquium on Automata, Languages and Programming (ICALP 1989), Lecture Notes in Computer Science, Springer-Verlag, Berlin, Vol. 372, pp. 687-703.

[5] Borodin, A. e El-Yaniv, R., Online Computation and Competitive Analysis, Cambridge University Press, 1998.

[6] Sutton, R. S. e Barto, A. G., Reinforcement Learning: An Introduction, The MIT Press, 1998. 
[7] Bertsekas, D. P. e Tsitsiklis, J. N., Neuro-dynamic Programming, Athena Scientific, 1996.

[8] Albers, S., Competitive On-Line Algorithms, BRICS Lecture Series, University of Aarhus, 1996. Disponível em: http://www.brics.dk/LS/96/2/BRICS-LS-96-2/BRICS-LS-96-2.html. Acessado em 06 de Abril de 2005.

[9] Bellman, R., Dynamic Programming, Princeton University Press, 1957.

[10] Watkins, C. J. C. H., Learning from Delayed Rewards, Phd thesis, University of Cambridge, 1989.

[11] Goldberg, D., Genetic Algorithms in Search, Optimization and Machine Learning, Addison-Wesley, Reading, MA, 1989.

[12] Hofri., M., Should the two headed disk be greedy?, Information Process Letters, 16:83-86, 1983.

[13] Flatto, L., Calderbank, A. R. e Coffman, E. G., Sequencing Problems in Two Server Systems, Mathematics of Operation Research, 10, pp. 585-598, 1985.

[14] Oliveira, S. P., O Problema dos k-Servos com Janela de Tempo, Dissertação de Mestrado, Instituto Militar de Engenharia, IME, Rio de Janeiro, 1996.

[15] Batta, R., e Mannur, N. R., Covering - Location Models for Emergency Situations that Require Multiple Servers Units, Man. Sci 36,16-23, 1990. 
[16] Charnes, A. e Storbeck, J. E., A Goal Programming Algorithm Model for the Siting of Multilevel SEM Systems, Socio-Economic Planning Science 14, 155-161, 1980.

[17] Chunch, R. L. e Eaton, D. J., A Bicriterion Maximal Covering Location Formulation which Considers the Satisfaction of Uncovered Demand, Decicio Sci. 22, 38-52, 1987.

[18] Marianov, V. e ReVelle, C., A Probabilistic Fire-Protection Siting Model with Joint Vehicle Reliability Requeriments, Journal of Regional Science, 71, 217-241, 1992.

[19] Belady, L. A., A Study of Replacement Algorithms for Virtual Storage Computers, IBM Systems Journal, IBM, pags. 78-101, 1966.

[20] Boyan, J. A. e Littman, M. L., Packet Routing in Dinamically Changing Networks: An Reinforcement Learning Approach. In Advances in Neural Information Processing Systems 6 (NIPS6), 671-678, 1994.

[21] Goldbarg, M. C. e Luna, H. P. C., Otimização Combinatória e Programação Linear: Modelos e Algoritmos, Editora Campus, 2000.

[22] Bartal, Y. e Koutsoupias, E., On the Competitive Ratio of the Work Function Algorithm for the k-Server Problem. Theor. Comput. Sci. 324(2-3): 337-345, 2004.

[23] Bartal, Y. e Grove, E., The Harmonic K-Server Algorithm is competitive. 23rd ACM Symposium on Theory of Computation, páginas 260-266, 1991. 
[24] Bartal, Y. e Rosen, A., The Distributed K-Server Problem: A Competitive Distributed Translator for K-Server Algorithms. Journal of Algorithms, v.23, n.2, p.241-264, Maio, 1997.

[25] Fiat, A., Rabani, Y., e Ravid, Y., Competitive K-Server Algorithms. Journal of Computer and System Sciences, 48:410-428, 1994.

[26] Koutsoupias, E. e Papadimitriou, C., On the K-Server Conjecture. Journal of the ACM, 42(5):971-983, 1995.

[27] Yao, A C. C., Probabilistic computations: Towards a unified measure of complexity, In: Proc. 17th Annual IEEE Symposium on Foundations of Computer Science, pags. 222-27, 1977.

[28] Jaakola, T., Jordan, M. I. e Singh, S. P., On the Convergence of Stochastic Iterative Dynamic Programming Algorithms, Neural Computation, vol 6, pp 1185-1201, 1994.

[29] Tarjan, R. E., Armotized Computational Complexity. SIAM Journal on Algebraic and Discrete Methods, 6:306-318, 1985.

[30] Koutsoupias, E., Online Algorithms and k-Server Conjecture. PhD thesis, University of California, San Diego, La Jolla, California, Junho, 1994.

[31] Belady, L. A., A Study of Replacement Algorithms for Virtual Storage Computers, IBM Systems Journal, IBM, pags. 78-101, 1966.

[32] Rocha, C. T. de M., Aloise, D., Aloise, D. J. e Melo, J. D., Heurísticas Paralelas de Busca em Vizinhança Variável para o Problema de Otimiza- 
ção do Emprego de Sondas de Produção Terrestre, XXXV SBPO, Natal, RN, 2003.

[33] Tesauro, G. J., TD-Gammon - A Self-Teaching Backgammon Achieves a Master-Level Play. Neural Computation, 6 (2):215-219. 